\title{
CHAOTIC VIBRATIONS \\ OF THE ONE-DIMENSIONAL WAVE EQUATION DUE TO A SELF-EXCITATION BOUNDARY CONDITION PART I: CONTROLLED HYSTERESIS
}

\author{
GOONG CHEN, SZE-BI HSU, JIANXIN ZHOU, AND WITH APPENDIX C BY \\ GUANRONG CHEN AND GIOVANNI CROSTA
}

\begin{abstract}
The study of nonlinear vibrations/oscillations in mechanical and electronic systems has always been an important research area. While important progress in the development of mathematical chaos theory has been made for finite dimensional second order nonlinear ODEs arising from nonlinear springs and electronic circuits, the state of understanding of chaotic vibrations for analogous infinite dimensional systems is still very incomplete.

The 1-dimensional vibrating string satisfying $w_{t t}-w_{x x}=0$ on the unit interval $x \in(0,1)$ is an infinite dimensional harmonic oscillator. Consider the boundary conditions: at the left end $x=0$, the string is fixed, while at the right end $x=1$, a nonlinear boundary condition $w_{x}=\alpha w_{t}-\beta w_{t}^{3}, \alpha, \beta>$ 0 , takes effect. This nonlinear boundary condition behaves like a van der Pol oscillator, causing the total energy to rise and fall within certain bounds regularly or irregularly. We formulate the problem into an equivalent first order hyperbolic system, and use the method of characteristics to derive a nonlinear reflection relation caused by the nonlinear boundary condition. Since the solution of the first order hyperbolic system depends completely on this nonlinear relation and its iterates, the problem is reduced to a discrete iteration problem of the type $u_{n+1}=F\left(u_{n}\right)$, where $F$ is the nonlinear reflection relation. We say that the PDE system is chaotic if the mapping $F$ is chaotic as an interval map. Algebraic, asymptotic and numerical techniques are developed to tackle the cubic nonlinearities. We then define a rotation number, following J.P. Keener [11], and obtain denseness of orbits and periodic points by either directly constructing a shift sequence or by applying results of M.I. Malkin [17] to determine the chaotic regime of $\alpha$ for the nonlinear reflection relation $F$, thereby rigorously proving chaos. Nonchaotic cases for other values of $\alpha$ are also classified. Such cases correspond to limit cycles in nonlinear second order ODEs. Numerical simulations of chaotic and nonchaotic vibrations are illustrated by computer graphics.
\end{abstract}

Received by the editors July 20, 1995 and, in revised form, October 16, 1996. 1991 Mathematics Subject Classification. Primary 35L05, 35L70, 58F39, 70L05.

The first and third authors' work was supported in part by NSF Grant DMS 9404380, Texas ARP Grant 010366-046, and Texas A\&M University Interdisciplinary Research Initiative IRI 96-39. Work completed while the first author was on sabbatical leave at the Institute of Applied Mathematics, National Tsing Hua University, Hsinchu 30043, Taiwan, R.O.C. The second author's work was supported in part by Grant NSC 83-0208-M-007-003 from the National Council of Science of the Republic of China. 


\section{INTRODUCTION}

The study of nonlinear vibrations/oscillations in mechanical and electronic systems has always been an important area of research by scientists and engineers [20]. In recent years, the primary emphasis of such research appears to be focused on the chaotic phenomena. Through numerical simulations, chaos has been shown to exist in many second order ordinary differential equations arising from nonlinear vibrating springs and electronic circuits, see [5], [18], for example. The mathematical justifications required in rigorously establishing the occurrence of chaos are technically very challenging. A number of such successful examples can be found in [8], [21]. While important progress in the development of mathematical chaos theory for nonlinear ordinary differential equations is being made, relatively little has been done in the mathematical study of chaotic vibrations in mechanical systems governed by partial differential equations (PDEs) containing nonlinearities. An exception is a pioneering paper by Holmes and Marsden [10], who have adapted Melnikov's approach to show the existence of chaos for an Euler-Bernoulli beam with certain distributed nonlinearity. Since solving that PDE numerically is somewhat involved, simulations were not given in [10], and thus so far we have not been able to "see" close up from [10] what spatio-temporal chaotic trajectories look like.

In general, the treatment of PDEs requires more sophisticated mathematical techniques. With the presence of nonlinearities, basic issues such as existence and uniqueness of solutions oftentimes are already quite difficult to settle, not to say the determination of chaotic behavior. Also, since PDEs have many different types, it is not easy to give a precise definition of chaos that is uniformly meaningful for a majority of PDEs. Thus, chaos for PDEs may have to be studied on a case-by-case basis.

Here, we wish to study chaotic vibrations of a vibrating string containing nonlinearities. Since nonlinearities may appear in many different ways, the first case - and perhaps the simplest one - we want to examine is a linear PDE but with a nonlinear boundary condition. We first recall the well-known van der Pol equation without forcing

$$
\begin{cases}\ddot{x}+\left(-\alpha \dot{x}+\beta \dot{x}^{3}\right)+\omega_{0}^{2} x=0 ; & \alpha, \beta, \omega_{0}^{2}>0, \\ x(0)=x_{0}, \quad \dot{x}(0)=x_{1}, & x_{0}, x_{1} \in \mathbf{R},\end{cases}
$$

where $\omega_{0}$ is the natural frequency of the corresponding linear harmonic oscillator. The time rate of change of energy, $E(t)=\frac{1}{2}\left[\dot{x}(t)^{2}+\omega_{0}^{2} x(t)^{2}\right]$, satisfies

$$
\begin{aligned}
\frac{d}{d t} E(t)= & \dot{x}(t)\left[\ddot{x}(t)+\omega_{0}^{2} x(t)\right] \\
= & \alpha \dot{x}^{2}(t)-\beta \dot{x}^{4}(t) \\
& \begin{cases}\geq 0, & \text { if }|\dot{x}(t)| \text { is small, } \\
\leq 0, & \text { if }|\dot{x}(t)| \text { is large. }\end{cases}
\end{aligned}
$$

Hence

$$
E(t) \text { is } \begin{cases}\text { increasing, } & \text { if }|\dot{x}(t)| \text { is small, } \\ \text { decreasing, } & \text { if }|\dot{x}(t)| \text { is large. }\end{cases}
$$

Therefore, unless the initial conditions satisfy $x_{0}=x_{1}=0$ in $(1.1)$, causing $E(t) \equiv 0$ for all $t>0$, we see that $E(t)$ eventually will rise and fall between a certain interval of the form $\left(B_{1}, B_{2}\right)$. This gives the name of "self-excited oscillations" for the 
van der Pol oscillator. The bounds $B_{1}$ and $B_{2}$ can be determined by "tuning" $\alpha$ and $\beta$, two of the design parameters in a vacuum tube or a nonlinear circuit. The self-regulating property (1.3) is highly useful in the design and application of servomechanisms in modern automatic control. The behavior of the rise and fall within a fixed bounded interval is obviously a precursor for chaos. However, Poincaré has shown that for the free van der Pol equation (1.1), the asymptotic trajectory of $(x(t), \dot{x}(t))$ on the phase plane tends to a limit cycle, see [20], for example. Therefore, the oscillation of the free van der Pol equation (1.1) is not chaotic. For chaos to happen, the van der Pol equation must be forced on the right-hand side, in the form

$$
\left\{\begin{array}{l}
\ddot{x}(t)+\left(-\alpha \dot{x}(t)+\beta \dot{x}^{3}(t)\right)+\omega_{0}^{2} x(t)=A \cos \omega t, \quad t \geq 0, \\
x(0)=x_{0}, \dot{x}(0)=x_{1},
\end{array}\right.
$$

when the parameters $\alpha, \beta$ and $A$ enter a certain regime; see [8].

The PDE analog of the linear simple harmonic oscillator is a vibrating string, whose displacement $w(x, t)$ at location $x$ at time $t$ satisfies the one dimensional wave equation

$$
\frac{\partial^{2} w(x, t)}{\partial t^{2}}-\frac{\partial^{2} w(x, t)}{\partial x^{2}}=0, \quad 0<x<1, \quad t>0
$$

(this equation also models acoustic wave propagation in a pipe) where we have normalized the string length to be one, and where the wave speed is also assumed to be one for simplicity. In the case when linear boundary conditions are imposed at the ends $x=0$ and $x=1,(1.5)$ is known to have infinitely many eigenmodes, each of which undergoes a simple harmonic motion. Thus, (1.5) is an infinite dimensional harmonic oscillator. At the left end $x=0$, assume that the string is fixed:

$$
w(0, t)=0, \quad t>0 .
$$

At the right end $x=1$, some force $f(t)$ is acting on the string:

$$
w_{x}(1, t)=f(t), \quad t>0 .
$$

This force $f(t)$ is now assumed to be of the nonlinear velocity feedback type: $f(t)=$ $\alpha w_{t}(1, t)-\beta w_{t}(1, t)^{3}, t>0$, yielding

$$
w_{x}(1, t)=\alpha w_{t}(1, t)-\beta w_{t}(1, t)^{3} ; \quad \alpha, \beta>0 .
$$

Note that this force $f$ has a double-well potential $V$ :

$$
V(X)=-\frac{1}{2} \alpha X^{2}+\frac{1}{4} \beta X^{4} ; \quad f=-d V(X) /\left.d X\right|_{X=w_{t}} .
$$

The energy of the wave equation (1.5) at time $t$ is given by

$$
E(t)=\frac{1}{2} \int_{0}^{1}\left[w_{x}(x, t)^{2}+w_{t}(x, t)^{2}\right] d x .
$$


Subject to the boundary conditions (1.6) and (1.7), the rate of change of energy is found to be

$$
\begin{aligned}
\frac{d}{d t} E(t) & =\int_{0}^{1}\left[w_{x}(x, t) w_{x t}(x, t)+w_{t}(x, t) w_{t t}(x, t)\right] d x \\
& =\left.w_{x}(x, t) w_{t}(x, t)\right|_{x=0} ^{1} \\
& =w_{x}(1, t) w_{t}(1, t) \\
& =\alpha w_{t}(1, t)^{2}-\beta w_{t}(1, t)^{4} \\
& \left\{\begin{array}{l}
\geq 0, \quad \text { if }\left|w_{t}(1, t)\right| \text { is small, } \\
\leq 0, \quad \text { if }\left|w_{t}(1, t)\right| \text { is large. }
\end{array}\right.
\end{aligned}
$$

Thus, the boundary condition (1.7) also causes the so-called "self-excited oscillation." Our study is motivated by the following:

(i) The qualitative analogy between (1.1) and (1.7) presents us with good nonlinear mathematical models which are quite useful as far as aiding the researcher in understanding the similarities and differences between the chaotic dynamics of ODEs and PDEs.

(ii) The use of nonlinear boundary conditions like (1.7) is in line with the contemporary theory of boundary control. The nonlinear effects are likely to aid in the development of "smart control" methodology/technology for today and the future.

(iii) The propagation of acoustic waves in a pipe satisfies the linear wave equation (1.5), while the nonlinear boundary condition (1.7) can be approximated and realized by analog, active devices in modern acoustics technology, leading the authors to believe that hardware may be developed/deployed in the near future which will allow the researcher to "hear" chaotic truly acoustical vibrations and control.

Other analogs of the nonlinear equation or boundary condition are:

(a) distributed "self-excited" nonlinear equation

$$
w_{t t}(x, t)-w_{x x}(x, t)=\alpha w_{t}(x, t)-\beta w_{t}(x, t)^{3} ; \quad \alpha, \beta>0
$$

where the nonlinearity is distributed over the entire spatial span, in lieu of the linear equation (1.5);

(b) nonlinear boundary condition of the form

$$
w_{x}(1, t)=\left[\alpha-\beta w^{2}(1, t)\right] w_{t}(1, t) ; \quad \alpha, \beta>0,
$$

in lieu of (1.7). (This is analogous to a second version of the van der Pol oscillator

$$
\left.\ddot{x}+\left(-\alpha+\beta x^{2}\right) \dot{x}+\omega_{0}^{2} x=0 .\right)
$$

Their treatments are more involved, and thus will be deferred to a future sequel.

For convenient future referencing, we compile the PDE system below:

$$
\begin{cases}w_{t t}(x, t)-w_{x x}(x, t)=0, & 0<x<1, t>0 \\ w(0, t)=0, & t>0 \\ w_{x}(1, t)=\alpha w_{t}(1, t)-\beta w_{t}(1, t)^{3}, & t>0 ; \alpha, \beta>0 \\ w(x, 0)=w_{0}(x), w_{t}(x, 0)=w_{1}(x), & 0 \leq x \leq 1\end{cases}
$$


where we may assume that the initial states $w_{0}$ and $w_{1}$ satisfy

$$
w_{0} \in C^{1}([0,1]), \quad w_{1} \in C^{0}([0,1]) .
$$

Relaxation of the regularity requirements in (1.11) can be made, as this becomes clear according to the subsequent development.

We use the method of characteristics to treat (1.10). By letting

$$
\left\{\begin{array}{l}
w_{x}(x, t)=u(x, t)+v(x, t), \\
w_{t}(x, t)=u(x, t)-v(x, t)
\end{array}\right.
$$

the PDE is diagonalized into a first order hyperbolic system

$$
\frac{\partial}{\partial t}\left[\begin{array}{l}
u(x, t) \\
v(x, t)
\end{array}\right]=\left[\begin{array}{cc}
1 & 0 \\
0 & -1
\end{array}\right] \frac{\partial}{\partial x}\left[\begin{array}{l}
u(x, t) \\
v(x, t)
\end{array}\right], 0<x<1, t>0 .
$$

The boundary condition at the left end $x=0, w(0, t)=0$, satisfies

$$
w_{t}(0, t)=0, \quad \forall t>0,
$$

or

$$
\begin{aligned}
& u(0, t)-v(0, t)=0, \\
& u(0, t)=v(0, t), \quad t>0,
\end{aligned}
$$

while at the right end $x=1$, we have

$$
u(1, t)+v(1, t)=\alpha[u(1, t)-v(1, t)]-\beta[u(1, t)-v(1, t)]^{3},
$$

or

$$
\beta[v(1, t)-v(1, t)]^{3}+(1-\alpha)[u(1, t)-v(1, t)]+2 v(1, t)=0, \quad t>0 .
$$

The initial conditions become

$$
\left\{\begin{array}{l}
u_{0}(x)=\frac{1}{2}\left[w_{0}^{\prime}(x)+w_{1}(x)\right] \in C([0,1]), \\
v_{0}(x)=\frac{1}{2}\left[w_{0}^{\prime}(x)-w_{1}(x)\right] \in C([0,1]) .
\end{array}\right.
$$

Equations (1.14)-(1.16) form the set of all initial-boundary data for the PDE (1.13).

From (1.13), since $u$ and $v$ satisfy, respectively,

$$
u_{t}-u_{x}=0, \quad v_{t}+v_{x}=0,
$$

we have the constancy along characteristics:

$$
\begin{aligned}
& u(x, t)=\text { constant, along } x+t=\text { constant, } \\
& v(x, t)=\text { constant, along } x-t=\text { constant. }
\end{aligned}
$$

For example, along a characteristic $x-t=\xi$ passing through the initial horizon $t=0$, we have

$$
v(x, t)=v_{0}(\xi), \quad \forall(x, t): \quad x-t=\xi, \quad 0<\xi<1 .
$$

When this characteristic intersects the right boundary $x=1$ at time $\tau$, we have

$$
v(1, \tau)=v_{0}(\xi) ; \quad \tau=1-\xi .
$$

At time $t=\tau$, a nonlinear reflection takes place according to (1.15):

$$
u(1, \tau)=F(v(1, \tau)) .
$$

The graph of this mapping, $\{(v(1, \tau), u(1, \tau)) \mid \tau>0\}$, after $v$ and $u$ are transposed, is a Poincaré section of the solution set $\mathcal{S} \equiv\{(u(x, t), v(x, t)) \mid 0 \leq x \leq 1$, $t>0\}$ of the PDE system (1.13)-(1.14). Furthermore, iterates of $F$ generate the 


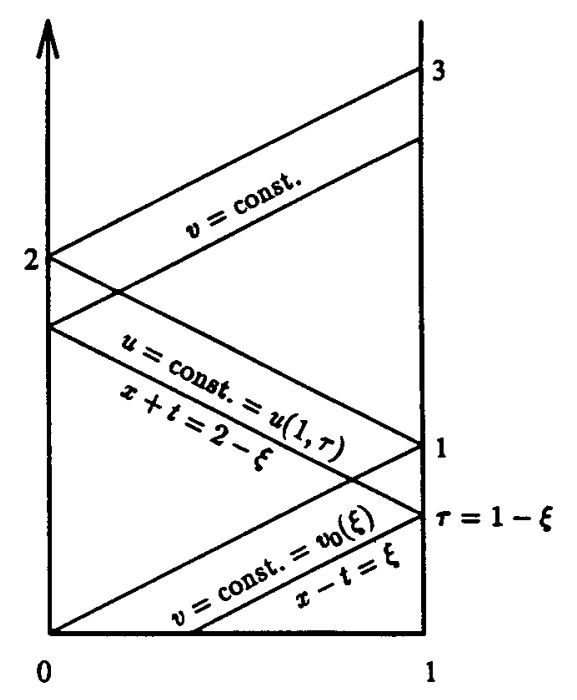

FiguRE 1. Reflection of characteristics

entire solution set $\mathcal{S}$ (see (6.1)and (6.2)). Therefore we say that chaotic vibration occurs if the Poincaré map $F$ is chaotic as an interval map; see (A.3). In (1.17), $u(1, \tau)$ is determined from $v(1, \tau)$ through solving the cubic equation (1.15). The relation (1.17) is obviously nonlinear. This observation alone is not enough - we must further recognize that the correspondence $F$ may not even be a single-valued mapping in general: For

$$
\beta(u-v)^{3}+(1-\alpha)(u-v)+2 v=0:
$$

(i) When $0<\alpha \leq 1$, for each given real value $v$, there exists a unique real solution $u$ satisfying (1.18). Thus $u=F(v)$ is well-defined.

(ii) When $\alpha>1$, there exists $v^{*}>0$, depending on $\alpha$ and $\beta$, s.t. for each real $v$ satisfying $|v|<v^{*}$, there exist three distinct real solutions $u$ satisfying (1.18). See (2.2)-(2.6) in $\S 2$. (But for $v \in \mathbf{R}$ satisfying $|v|>v^{*}$, there corresponds a unique real solution $u$ satisfying (1.18).)

The fact in (ii) above bluntly says that solutions to the hyperbolic system (1.13)(1.16), and consequently, to the original PDE system (1.10), are not unique when $\alpha>1$.

In handling the multivalued relation (1.18), it is natural to expect that relaxation oscillations should also happen, because the curve representing (1.18) is qualitatively similar to the ODE case; compare our Fig. 5(a) to [20, Fig. 6.1, p. 137]. Therefore a natural hysteresis loop should also be constructible from (1.18), even though no mechanical experiments have been actually performed to confirm this; see Fig. 6(b) later. If this natural hysteresis loop is adopted as the reflection relation $u=F(v)$, then obviously uniqueness of solutions of the PDE system (1.13)-(1.16) will come as a consequence. At this moment of writing, we have made certain progress in studying the chaotic vibration if $u=F(v)$ is assumed to be the natural hysteresis loop; see a forthcoming paper elsewhere.

In this paper, to handle the nonuniqueness of $u$ for $|v|<v^{*}$ when $\alpha>1$, we will choose (out of three branches of $u$-solutions) a single branch as the solution 
TABLE 1. Classification of chaotic/non-chaotic behavior of $F$ according to $\alpha$

\begin{tabular}{|l|l|}
\hline range of $\alpha$ & behavior of $F$ \\
\hline $0<\alpha \leq 1$ & $\begin{array}{l}\text { The hysteresis curve is single-valued and } \\
\text { continuous. } F \text { has a globally attracting period-2 } \\
\text { orbit. }\end{array}$ \\
\hline $1<\alpha<2.9312$ & $\begin{array}{l}\text { (The controlled hysteresis curve becomes discontinuous } \\
\text { for } \alpha>1 \text {.) A globally attracting period-2 orbit. }\end{array}$ \\
\hline $2.9312<\alpha<4$ & $\begin{array}{l}\text { A unique period-2 orbit that attracts almost } \\
\text { every point. (For } 3.7444 \cdots<\alpha<4, \text { there is "transient } \\
\text { chaos" that fades in time.) }\end{array}$ \\
\hline $4<\alpha<7$ & Chaotic with a single strange attractor. \\
\hline $7<\alpha<\infty$ & Chaotic with two disconnected strange attractors. \\
\hline
\end{tabular}

for $u=F(v)$; see Fig. 7 later. Physically speaking, such a branch can be chosen only with artificial intervention or special engineering design, for, unlike the natural hysteresis loop, this branch is not stable, so instability prevents it from naturally occurring. A plausible electronic analog circuit realization is offered in Appendix B. Nevertheless, after this choice the nonlinear equation (1.18) (and consequently, (1.7) and (1.15) as well) is indeed satisfied and the solution uniqueness is effected that is totally mathematically acceptable and justified. The correspondence

$$
u(1, \tau)=F(v(1, \tau)), \quad \tau>0, \quad \text { see }(2.22),
$$

becomes single-valued and is a well-defined functional relation. We call (1.19) the controlled hysteretic reflection relation. Now that the reflection becomes welldefined at the boundary $x=1$, by the method of characteristics, reflections at both $x=0$ and $x=1$ can be carried on indefinitely, as shown in Fig. 1, enabling the construction of the solution $(u(x, t), v(x, t))$ at every point $(x, t): 0 \leq x \leq 1, t \geq 0$. From now on, whenever we refer to the solution of (1.13)-(1.16), we mean that the solution satisfies the hysteretic reflection relation (1.19) at $x=1$.

For the behavior of iterates of a piecewise continuous interval map $u=f(v)$, earlier interesting results may be found in Lasota and Yorke [12] and Li and Yorke [14]. But we should credit J.P. Keener for some fundamental contributions to the analysis of chaos for such maps in [11], where he treated problems arising from mathematical biology, leading to a difference equation $u_{n+1}=F\left(u_{n}\right)$ with piecewise continuous $F$. The situation of piecewise continuity of $F$ in our paper is quite similar to Keener's, and we have found his analysis of high significance to our study. However, Keener's major results in [11], concerned with what he has termed the "non-overlapping" case, are not applicable to our work here. Even for Keener's "overlapping" case [11, §4], our situations differ from his in significant details and varieties, and thus his theorem there is only applicable to very special cases of ours. We need to refine Keener's arguments (in his theorems) considerably so as to work for our cases here, with the greatest challenge lying in the quantification of the inverse cubic map $F$ containing jump discontinuities, in order to construct a shift sequence of intervals. For large $\alpha$, the construction of shift sequences becomes out of reach. We need to resort to some more recent techniques and results of M.E. Malkin $[16,17]$ for our purpose. 
The summary for our findings is given in Table 1. The visualization of chaotic behavior of solutions is illustrated by computer graphics in $\S 6$.

Several other types of chaotic vibrations of the one-dimensional wave equation of different physical origins have also been identified by us recently. We hope to report them in the sequel. For other recent progress, see Chen, Hsu and Zhou [3].

\section{Cardan's Formula and the Hysteretic Reflection Curve}

Consider the cubic equation (1.18). We write it as

$$
x^{3}+p x+q=0,
$$

where

$$
x=u-v, p=\frac{1-\alpha}{\beta}, q=\frac{2 v}{\beta} .
$$

Let

$$
\mathcal{D}=\frac{p^{3}}{27}+\frac{q^{2}}{4}
$$

This is the discriminant in Cardan's formula for solutions of the cubic equation (2.1). It is well known that

(i) When $\mathcal{D}>0$, there is only one real solution $y$, of (2.1), expressed by

$$
y=\sqrt[3]{-\frac{q}{2}+\sqrt{\mathcal{D}}}+\sqrt[3]{-\frac{q}{2}-\sqrt{\mathcal{D}}}
$$

(ii) When $\mathcal{D}<0$, there exist three distinct real solutions $x_{1}, x_{2}$ and $x_{3}$ of $(2.1)$, expressible as

$$
\begin{aligned}
& x_{1}=\sqrt[3]{-\frac{q}{2}+\sqrt{\mathcal{D}}}+\sqrt[3]{-\frac{q}{2}-\sqrt{\mathcal{D}}}, \\
& x_{2}=\omega \sqrt[3]{-\frac{q}{2}+\sqrt{\mathcal{D}}}+\bar{\omega} \sqrt[3]{-\frac{q}{2}-\sqrt{\mathcal{D}}} \\
& x_{3}=\bar{\omega} \sqrt[3]{-\frac{q}{2}+\sqrt{\mathcal{D}}}+\omega \sqrt[3]{-\frac{q}{2}-\sqrt{\mathcal{D}}},
\end{aligned}
$$

where $\omega=\frac{1}{2}(-1+\sqrt{3} i)$ is a cubic root of unity and $\bar{\omega}$ is the complex conjugate of $\omega$. An alternative way of representations is, for $k=1,2,3$,

$$
x_{k}=2 S \cos \frac{\phi+2 k \pi}{3} ; S \equiv \sqrt{-\frac{p}{3}}, \phi=\cos ^{-1}\left(-\frac{\sqrt{27} q}{2 \sqrt{-p^{3}}}\right) ;
$$

(iii) When $\mathcal{D}=0$, there exist three real solutions, two of which are identical:

$$
x_{1}=2 \sqrt[3]{-\frac{q}{2}}, \quad x_{2}=x_{3}=\sqrt[3]{\frac{q}{2}}
$$

From the above solutions of Cardan, we are now in a position to determine the hysteretic reflection relation $F$ in (1.17). The situation is simplest if

$$
0<\alpha<1 \text {, }
$$

because this gives

$$
\mathcal{D}=\frac{1}{27}\left(\frac{1-\alpha}{\beta}\right)^{3}+\frac{1}{4}\left(\frac{4 v^{2}}{\beta^{2}}\right)>0, \quad \forall v \in \mathbf{R} .
$$




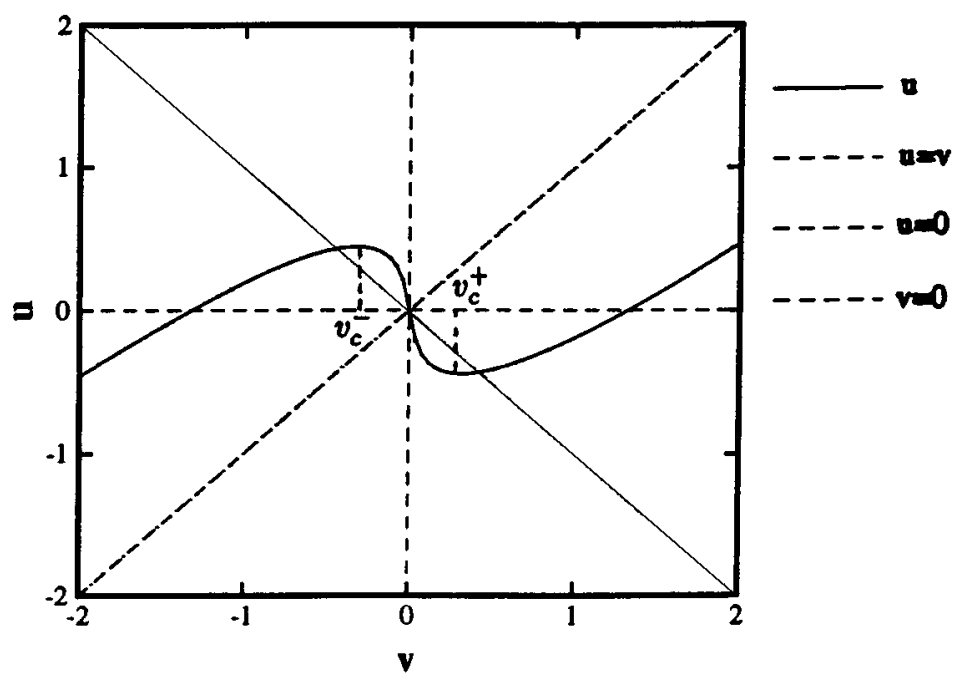

Figure 2. The curve $u=F(v)$, with $\alpha=3 / 4, \beta=1$.

Thus, for each $v \neq 0$, there exists a unique $u$, given by

$$
u=v+x_{1}
$$

$$
=v+\sqrt[3]{-\frac{v}{\beta}+\sqrt{\frac{(1-\alpha)^{3}}{27 \beta^{3}}+\frac{v^{2}}{\beta^{2}}}}+\sqrt[3]{-\frac{v}{\beta}-\sqrt{\frac{(1-\alpha)^{3}}{27 \beta^{3}}+\frac{v^{2}}{\beta^{2}}}} .
$$

When $\alpha=1$, the situation is similar. Therefore, we obtain

$$
u=F(v) \equiv \text { RHS of (2.11), } \forall v \in \mathbf{R}, \forall \alpha: 0<\alpha \leq 1 .
$$

$F$ is easily checked to be an odd function. Plots of $F$ are illustrated in Fig. 2, for $\alpha=3 / 4$, and $\beta=1$.

For easy visual guide and analysis needs, let us define the function

$$
\begin{aligned}
& f(x, v)=\beta x^{3}+(1-\alpha) x+2 v, \\
& \alpha>0, \beta>0, x \in \mathbf{R}, v \in \mathbf{R} .
\end{aligned}
$$

When $\alpha: 0<\alpha \leq 1$, the case considered by us in (2.9)-(2.12) for any given fixed $v \in \mathbf{R}$, the curve of $z=f(x, v)$ does not have any local maximum or minimum with respect to $x$; it is monotonically increasing with respect to $x$ and intersects the $x$-axis at a unique point $\bar{x}$, with $\bar{x}$ varying with respect to $v$. Writing $\bar{x}=\bar{x}(v)$, we obviously have

$$
\begin{aligned}
& u-v=\bar{x}(v), \\
& u=v+\bar{x}(v)=F(v)=\text { RHS of (2.11), shown in Fig. } 3 .
\end{aligned}
$$

Next, we consider $\alpha: \alpha>1$. For any given fixed $v \in \mathbf{R}$, the curve $z=f(x, v)$ now has a local maximum and a local minimum happening at $x=-x^{*}$ and $x=x^{*}$, 


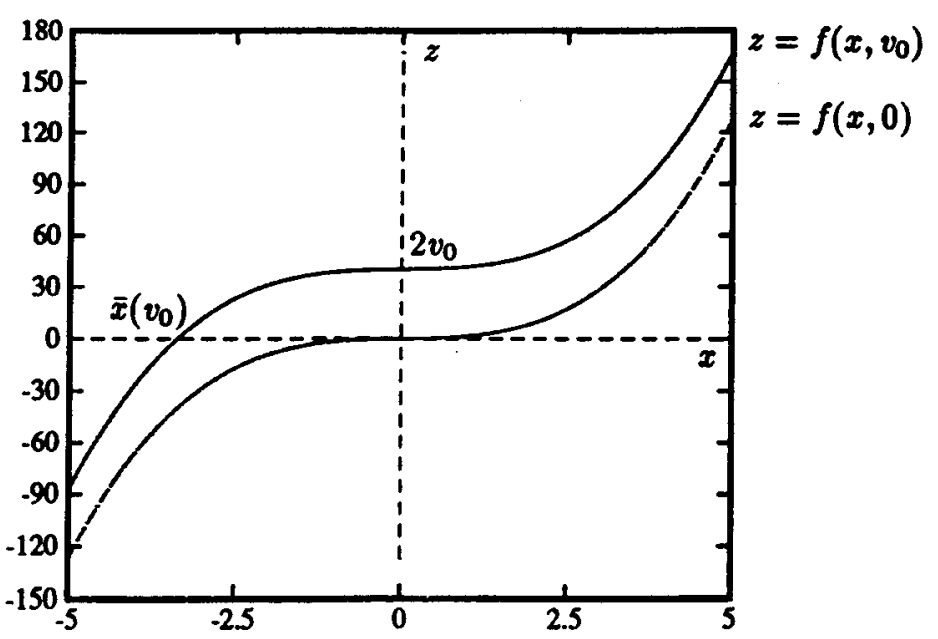

Figure 3 . The curve $z=f(x, v)$, for $0<\alpha \leq 1, \beta>0$.

respectively, where

$$
\frac{\partial f\left( \pm x^{*}, v\right)}{\partial x}=\left.\left[3 \beta x^{2}+(1-\alpha)\right]\right|_{x= \pm x^{*}}=0 .
$$

We get

$$
x^{*}=\sqrt{\frac{\alpha-1}{3 \beta}},
$$

independent of $v$. The curve $z=f(x, v)$ may intersect the $x$-axis at three, two, or one point, as shown in Fig. 4(a), (b) and (c).

Solving $v$ from

$$
f\left(-x^{*}, v\right)=0, \quad \text { or } \quad f\left(x^{*}, v\right)=0,
$$

we obtain

$$
v^{*}=\frac{\alpha-1}{3} \sqrt{\frac{\alpha-1}{3 \beta}}, \quad \alpha>1, \beta>0,
$$

satisfying

$$
f\left(-x^{*},-v^{*}\right)=0, f\left(x^{*}, v^{*}\right)=0 .
$$

Thus, we get that

$$
z=f(x, v) \text { intersects the } x \text {-axis at }\left\{\begin{array}{l}
3 \text { points, if }|v|<v^{*} \\
2 \text { points, if } v=-v^{*} \text { or } v=v^{*} \\
1 \text { point, if }|v|>v^{*}
\end{array}\right.
$$

Now, for $\alpha>1$, we use Cardan's formula. For the discriminant $\mathcal{D}$ in (2.3), we have

$$
\mathcal{D}\left\{\begin{array}{lll}
<0, & \text { if } & |v|<v^{*} \\
=0, & \text { if } & v= \pm v^{*} \\
>0, & \text { if } & |v|>v^{*}
\end{array}\right.
$$


(a)
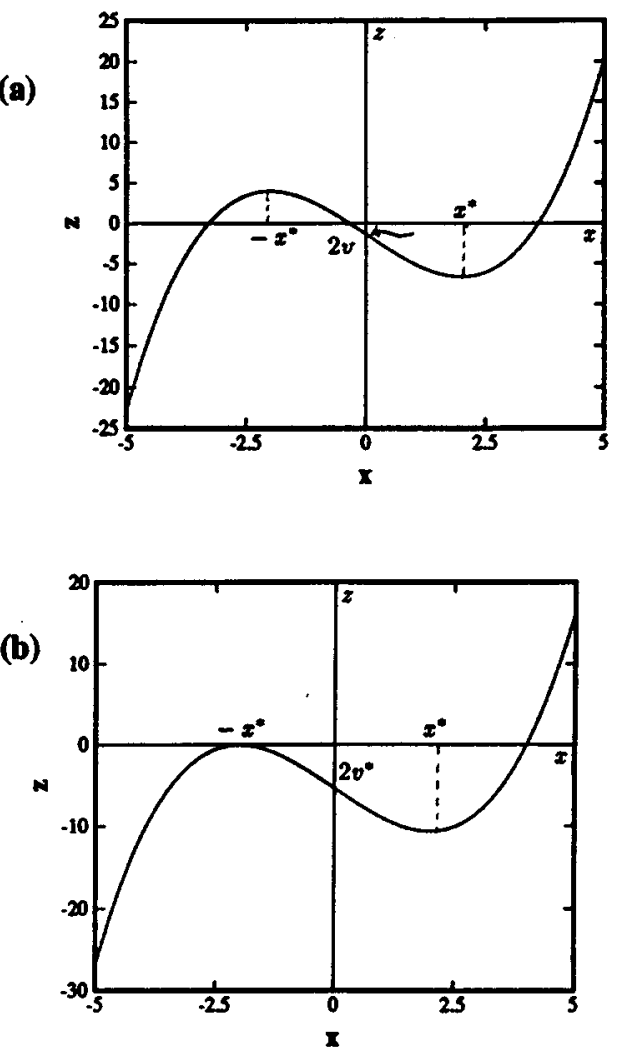

(c)

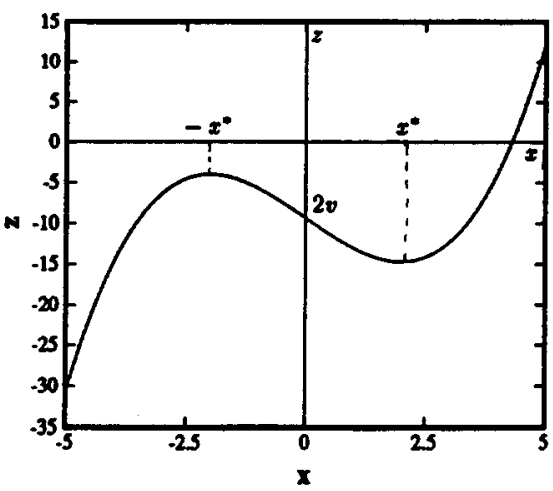

Figure 4. For $\alpha>1, \beta>0$, the curve $z=f(x, v)$, intersects the $x$-axis at (a) three points, if $|v|<v^{*}$; (b) two points, if $|v|=v^{*}$, and (c) one-point, if $|v|>v^{*}$.

Therefore, the hysteretic reflection relation is piecewise defined for $v:|v|>v^{*}$ in the following way:

$$
u=F(v)=v+\sqrt[3]{-\frac{v}{\beta}+\sqrt{-\frac{(\alpha-1)^{3}}{27 \beta^{3}}+\frac{v^{2}}{\beta^{2}}}}+\sqrt[3]{-\frac{v}{\beta}-\sqrt{-\frac{(\alpha-1)^{3}}{27 \beta^{3}}+\frac{v^{2}}{\beta^{2}}}} .
$$


When $|v|<v^{*}$, we have $\mathcal{D}<0$. Therefore, by $(2.5)-(2.6)$,

$$
u-v=\bar{x}_{k}, k=1,2,3, \quad \text { for } \quad|v|<v^{*} .
$$

These three values $\bar{x}_{1}, \bar{x}_{2}$ and $\bar{x}_{3}$ are the intersection points of the curve $z=f(x, v)$ with the $x$-axis as shown in Fig. 4(a). Thus, for each value of $v$, there correspond three distinct values of $u: u=v+\bar{x}_{k}, k=1,2,3$. See Fig. 5(a) for such multiplevalued relations, with $\alpha=4, \beta=1$. For problem (1.10), and consequently for (1.13)-(1.16), we expect a unique physical solution to exist. As indicated in $\S 1$, this physical solution should be a relaxation oscillation, i.e., one which moves only along the stable branch, forming a natural hysteresis loop; see Fig. 5(b). In the mathematical treatment of this paper, we pick a different (controlled) branch in order to have uniqueness. The choice we make is:

$$
\begin{aligned}
& u=F(v) \equiv v+\bar{x}_{1} \\
&=v+\bar{\omega} \sqrt[3]{-\frac{v}{\beta}+\sqrt{\frac{(1-\alpha)^{3}}{27 \beta^{3}}+\frac{v^{2}}{\beta^{2}}}}+\omega \sqrt[3]{-\frac{v}{\beta}-\sqrt{\frac{(1-\alpha)^{3}}{27 \beta^{3}}+\frac{v^{2}}{\beta^{2}}}} \\
& \quad \text { for }|v|<v^{*}, \bar{x}_{1}=\text { the root of } f(x, v)=0 \text { given by (2.5). }
\end{aligned}
$$

This branch and the ensuing $F$ have the following advantages:

(1) it is skew-symmetric and is more mathematically tractable;

(2) in principle, it can be realized through a nonlinear circuit design; see Appen$\operatorname{dix} \mathrm{C}$

(3) the resulting mathematical solution remains a weak solution of the PDE system (1.10).

(Incidentally, we might add that different choices of piecewise curves from the three branches along with many jump discontinuities to make up $F$ are all mathematically legitimate. But they may run into a severe trouble with mathematical tractability.) Therefore, the hysteretic reflection relation $u=F(v)$ is now defined for $|v|>v^{*}$ and $|v|<v^{*}$ through (2.20) and (2.22), respectively. It has jump discontinuities at $v= \pm v^{*}$. At the points $v= \pm v^{*}$, the left-hand and right-hand limits of $F$ exist. We define the value of $F$ at $v=v^{*}$ (respectively, $-v^{*}$ ) to be the right-hand (respectively, left-hand) limit of $F$. Such pointwise values at $v= \pm v^{*}$ will not affect the subsequent development. Thus, now $u=F(v)$ is defined for all $v \in \mathbf{R}$. For an illustration, see Fig. 6 .

From now on, we use $f^{n}(x) \equiv f \circ f \circ \cdots \circ f(x)$ ( $n$ times) to denote the iterated composition map of a function $f$, and use $f(x)^{n}$ to denote the $n$-th power of the map $f(x)$. For the iterated map $F^{n}(x)$, we have the following basic property.

Theorem 2.1. Let $\alpha, \beta>0$. Let $u=F(v)$ be the hysteretic reflection relation as defined above. Then there exists a $B>0$ depending only on $\alpha$ and $\beta$ s.t. for any $v_{0} \in \mathbf{R}$, there exists an $N\left(v_{0}\right) \in \mathbf{Z}^{+} \equiv\{0,1,2, \cdots\}$ satisfying

$$
\left|F^{n}\left(v_{0}\right)\right| \leq B, \quad \forall n \in \mathbf{Z}^{+}, n \geq N\left(v_{0}\right) .
$$

Proof. Use the recurrence relation between $F^{n}$ and $F^{n+1}$. 


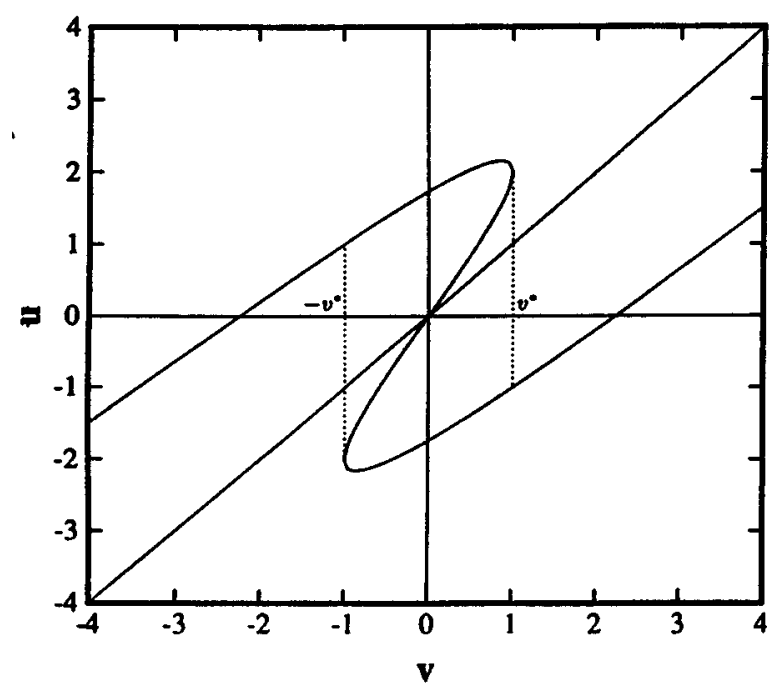

(a)

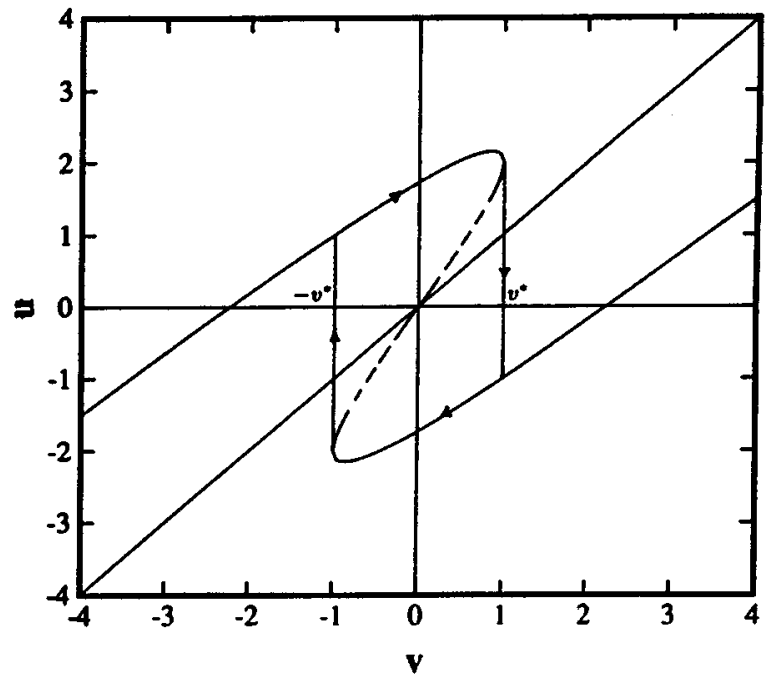

(b)

Figure 5. (a) The multiple-valued relation $u=F(v)$, with $\alpha=$ $4, \beta=1$. (b) The corresponding natural hysteresis loop.

The sharpest bound $B$ for Theorem 2.1 may be determined directly from the invariant regions of the iterated map $F^{n}(\cdot)$. This will be discussed in the next section. 


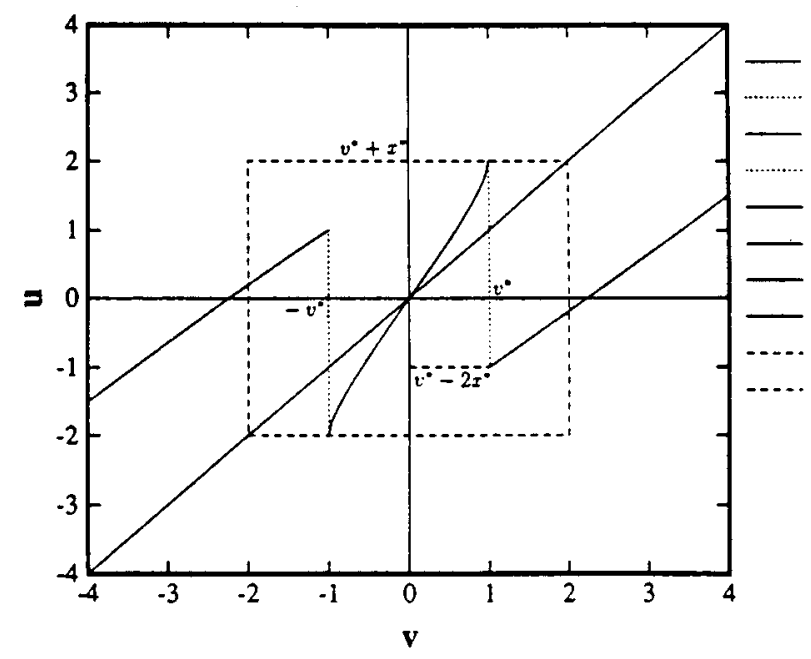

Figure 6 . The single-valued controlled hysteresis relation $u=$ $F(v)$, with $\alpha=4, \beta=1$.

\section{Important Properties of the Hysteretic Reflection Curve}

The nonlinear asymptotic behavior of the solution $(u(x, t), v(x, t))$ is determined by the hysteretic reflection curve, whose major properties are characterized below. We divide the discussion into several cases.

3.1. The continuous reflection curve: $\mathbf{0}<\boldsymbol{\alpha} \leq \mathbf{1}$. As noted in (2.8)-(2.11), when $0<\alpha \leq 1$, for each given $v \in \mathbf{R}$, there exists a unique solution $u \in \mathbf{R}$ of

$$
\beta(u-v)^{3}+(1-\alpha)(u-v)+2 v=0 .
$$

With $f(x, v)$ being defined as in (2.13), we rewrite (3.1) as

$$
f(u-v, v)=0,
$$

or

$$
f(g(v), v)=0, \quad g(v) \equiv u-v,
$$

where $g(v)=x$ is the unique real solution of $f(x, v)=0$. Although $g(v)$ admits an explicit representation afforded by (2.11), in our discussions to follow, we have found it convenient to manipulate $g(v)$ at the implicit level.

We list, itemwise, useful properties of $g(v)$ and $F(v): u=F(v)=v+g(v)$ in the following.

(P.1) $g$ is odd: $g(-v)=-g(v) ; g(v)<0$ if $v>0$.

(P.2) $g$ is strictly decreasing:

$$
\begin{aligned}
& \frac{d}{d v} f(g(v), v)=0, \\
& \frac{\partial f(g(v), v)}{\partial x} \cdot g^{\prime}(v)+\frac{\partial f(g(v), v)}{\partial v}=0 .
\end{aligned}
$$


Hence

$$
g^{\prime}(v)=\left.\frac{-\frac{\partial f}{\partial v}}{\frac{\partial f}{\partial x}}\right|_{x=g(v)}=\frac{-2}{3 \beta(g(v))^{2}+(1-\alpha)}<0 .
$$

In particular,

$$
g^{\prime}(0)=\frac{-2}{1-\alpha}<0
$$

(P.3) $F$ is an odd function, $F(0)=0$, and 0 is a repelling fixed point:

$$
\begin{aligned}
F^{\prime}(0) & =1+g^{\prime}(0) \\
& =1-\frac{2}{1-\alpha}=-\frac{1+\alpha}{1-\alpha},
\end{aligned}
$$

so $\left|F^{\prime}(0)\right|>1$.

(P.4) The mapping $u=F(v)$ does not have any other fixed points than $v=0$ :

If $\bar{v}=F(\bar{v})=\bar{v}+g(\bar{v})$, then $g(\bar{v})=0$. By (P.2) and the fact that $g(0)=0$, we get $\bar{v}=0$.

(P.5) The critical points of the function $u=F(v)$ happen at $v_{c}^{+}$and $v_{c}^{-}$s.t.

$$
\begin{aligned}
& \qquad g\left(v_{c}^{ \pm}\right)= \pm \sqrt{\frac{1+\alpha}{3 \beta}}: \\
& F^{\prime}\left(v_{c}^{ \pm}\right)=1+g^{\prime}\left(v_{c}^{ \pm}\right)=1-\frac{2}{3 \beta\left(g\left(v_{c}^{ \pm}\right)\right)^{2}+(1-\alpha)}=0 \quad \text { (by (3.4)). } \\
& 3 \beta\left(g\left(v_{c}^{ \pm}\right)\right)^{2}+(1-\alpha)=2, \\
& \text { and so }
\end{aligned}
$$

$$
g\left(v_{c}^{ \pm}\right)= \pm \sqrt{\frac{1+\alpha}{3 \beta}} .
$$

(P.6) Besides $v=0, u=F^{2}(v)$ has two fixed points $v_{p 2}^{+}$and $v_{p 2}^{-}$s.t.

$$
g\left(v_{p 2}^{ \pm}\right)= \pm \sqrt{\frac{\alpha}{\beta}} .
$$

(The subscript " $p 2$ " means "period 2"; see (3.8) shortly.) Let

$$
\begin{aligned}
\tilde{v} & =F^{2}(\tilde{v})=F(F(\tilde{v}))=F(\tilde{v})+g(F(\tilde{v})) \\
& =[\tilde{v}+g(\tilde{v})]+g(F(\tilde{v})) .
\end{aligned}
$$

Thus

$$
\begin{aligned}
& g(\tilde{v})+g(F(\tilde{v}))=0, \\
& g(-\tilde{v})=-g(\tilde{v})=g(F(\tilde{v})) .
\end{aligned}
$$

But by (P.2), $g$ is 1-1. Hence

$$
\begin{aligned}
-\tilde{v} & =F(\tilde{v})=\tilde{v}+g(\tilde{v}), \\
g(\tilde{v}) & =-2 \tilde{v}=\beta[g(\tilde{v})]^{3}+(1-\alpha) g(\tilde{v}) .
\end{aligned}
$$

Therefore

$$
g(\tilde{v})\left[g(\tilde{v})-\sqrt{\frac{\alpha}{\beta}}\right]\left[g(\tilde{v})+\sqrt{\frac{\alpha}{\beta}}\right]=0
$$


Writing $\pm \tilde{v}=v_{p 2}^{ \pm}$, we get

$$
g\left(v_{p 2}^{ \pm}\right)= \pm \sqrt{\frac{\alpha}{\beta}} .
$$

(P.7) There exist two points $v_{S 1}^{+}$and $v_{S 1}^{-}$s.t.

$$
F^{\prime}\left(v_{S 1}^{ \pm}\right)=-1, \quad g\left(v_{S 1}^{ \pm}\right)= \pm \sqrt{\frac{\alpha}{3 \beta}}
$$

On the other hand, we have $F^{\prime}(v)<1$ for all $v \in \mathbf{R}$, and consequently, there do not exist any points $v$ satisfying $F^{\prime}(v)=1$ :

First

$$
F^{\prime}(v)=1+g^{\prime}(v)=1-\frac{2}{3 \beta(g(v))^{2}+(1-\alpha)} .
$$

Let $\tilde{v}$ satisfy $F^{\prime}(\tilde{v})=-1$. Then from (3.7),

$$
\begin{gathered}
3 \beta(g(\tilde{v}))^{2}+(1-\alpha)=1 \\
g(\tilde{v})^{2}=\frac{\alpha}{3 \beta} .
\end{gathered}
$$

Writing $\pm \tilde{v}=v_{S 1}^{ \pm}$, we get (3.6). From (3.7), it is obvious that $F^{\prime}(v)=$ $1+g^{\prime}(v)<1$, because $g^{\prime}(v)<0$, for all $v \in \mathbf{R}$.

The two points $v_{p 2}^{+}$and $v_{p 2}^{-}$in (P.6) are obviously of period two:

$$
F\left(v_{p 2}^{+}\right)=v_{p 2}^{-}, \quad F\left(v_{p 2}^{-}\right)=v_{p 2}^{+} .
$$

At $v=v_{p 2}^{+}$and $v_{p 2}^{-}$, the curve $u=F(v)$ intersects the straight line $u+v=$ 0 . Using the above listed properties (P.1)-(P.7), we can now provide the important stability analysis of $v_{p 2}^{ \pm}$.

Theorem 3.1. Let $0<\alpha \leq 1$. The map $F^{2}(\cdot)$ has three fixed points: $0, v_{p 2}^{+}$and $v_{p 2}^{-}$. The origin $v=0$ (with period-1 only) is a repelling fixed point:

$$
\left|\left[F^{2}(0)\right]^{\prime}\right|>1
$$

while the two fixed points $v_{p 2}^{+}$and $v_{p 2}^{-}$satisfy

$$
\left|\left[F^{2}\left(v_{p 2}^{ \pm}\right)\right]^{\prime}\right|<1
$$

and $v_{p 2}^{+}$and $v_{p 2}^{-}$having period-2 are globally attracting s.t., for $v \neq 0$, either

$$
\lim _{n \rightarrow \infty} F^{2 n}(v)=v_{p 2}^{+}, \quad \lim _{n \rightarrow \infty} F^{2 n+1}(v)=v_{p 2}^{-},
$$

or

$$
\lim _{n \rightarrow \infty} F^{2 n}(v)=v_{p 2}^{-}, \quad \lim _{n \rightarrow \infty} F^{2 n+1}(v)=v_{p 2}^{+} .
$$

Proof. We first note that (3.9) is a consequence of (P.3). Differentiating $F^{2}$ :

$$
\left[F^{2}(v)\right]^{\prime}=F^{\prime}(F(v)) F^{\prime}(v),
$$

at $v=v_{p 2}^{ \pm}$, we get

$$
\begin{aligned}
{\left[F^{2}\left(v_{p 2}^{ \pm}\right)\right]^{\prime} } & =F^{\prime}\left(F\left(v_{p 2}^{ \pm}\right)\right) F^{\prime}\left(v_{p 2}^{ \pm}\right) \\
& =F^{\prime}\left(v_{p 2}^{\mp}\right) \cdot F^{\prime}\left(v_{p 2}^{ \pm}\right), \quad \text { by (3.8). }
\end{aligned}
$$


When $0<\alpha<1 / 2$, we have

$$
\sqrt{\frac{\alpha}{3 \beta}}<\sqrt{\frac{\alpha}{\beta}}<\sqrt{\frac{1+\alpha}{3 \beta}} ;
$$

by (P.1), (P.2), (P.5)-(P.7), we obtain

$$
v_{S 1}^{-}>v_{p 2}^{-}>v_{c}^{-},
$$

or equivalently,

$$
v_{S 1}^{+}<v_{p 2}^{+}<v_{c}^{+} .
$$

From (3.7), (3.14), (P.1) and (P.2), we deduce that

$$
-1<F^{\prime}\left(v_{p 2}^{+}\right)<0
$$

Similarly, by the oddness of $F(\cdot)$, we get

$$
0<F^{\prime}\left(v_{p 2}^{-}\right)<-1
$$

Using (3.15) and (3.16) in (3.13), we obtain

$$
\left|\left[F^{2}\left(v_{p 2}^{ \pm}\right)\right]^{\prime}\right|<1 .
$$

Next, we consider the case $1 / 2<\alpha \leq 1$. Here we have

implying, similarly as before,

$$
\sqrt{\frac{\alpha}{3 \beta}}<\sqrt{\frac{1+\alpha}{3 \beta}}<\sqrt{\frac{\alpha}{\beta}},
$$

$$
v_{S 1}^{+}<v_{c}^{+}<v_{p 2}^{+} .
$$

See Fig. 2 for the graph of $F$ with $\alpha=0.75, \beta=1$. But $v_{p 2}^{+}<v_{c}^{+}$implies $g\left(v_{p 2}^{+}\right)>$ $g\left(v_{c}^{+}\right)$and thus, from (3.7), (P.1) and (P.7), we have

$$
0<F^{\prime}\left(v_{p 2}^{+}\right)<1 \text {. }
$$

Similarly,

$$
0<F^{\prime}\left(v_{p 2}^{-}\right)<1 .
$$

From (3.13), (3.18) and (3.19), we again have (3.17).

When $\alpha=1 / 2$, we have

$$
\sqrt{\frac{1+\alpha}{3 \beta}}=\sqrt{\frac{\alpha}{\beta}}
$$

and thus,

$$
v_{p 2}^{ \pm}=v_{c}^{ \pm}
$$

giving

$$
\left[F\left(v_{p 2}^{ \pm}\right)\right]^{\prime}=\left[F\left(v_{c}^{ \pm}\right)\right]^{\prime}=0
$$

so (3.17) again holds.

It remains to establish (3.11) and (3.12). By Theorem $2.1, F^{2 n}(v)$ will eventually be bounded by $B$ : there exists $N(v) \in \mathbf{Z}^{+}$s.t.

$$
\left|F^{2 n}(v)\right| \leq B, \quad \forall n \geq N(v) .
$$

Therefore we need only consider all $v \in[-B, B]$, where $B$ is sufficiently large and $B>v_{p 2}^{+}$. But on the interval $[-B, B], v_{p 2}^{+}$and $v_{p 2}^{-}$are the two unique attracting 
fixed points of $F^{2}$. By the oddness of $F$, and hence $F^{2}(\cdot), F^{2 n}(\cdot)$ and $F^{2 n+1}(\cdot)$, and by (3.8) and the fact that $F$ alternates signs in a small neighborhood of $v_{p 2}^{-}$and in that of $v_{p 2}^{+}$, we see that all iterates $F^{2 n}(v)$ and $F^{2 n+1}(v)$ will be attracted to $v_{p 2}^{ \pm}$ either as in (3.11) or in (3.12).

The number $\alpha=1$, which forms the divide between the continuous $F$ in this subsection and the discontinuous $F$ in the following subsection, has some particular significance. When $\beta=0$ in (1.15), the linear system (1.13)-(1.16) is known to be time irreversible because the corresponding evolution semigroup is nilpotent; see Chen and Zhou [4, pp. 243-246]. A higher dimensional case is studied in Majda [15].

3.2. The discontinuous hysteretic reflection curve $\boldsymbol{\alpha}>\mathbf{1}$. As noted in $\S 2$, when $\alpha>1$, the hysteretic reflection curve $u=F(v)$ consists of three continuous pieces: $F=F_{1} \cup F_{2} \cup F_{3}$. The relative positioning and the magnitudes of discontinuities between the curves of $F_{1}, F_{2}$, and $F_{3}$ are crucial in the analysis of the chaotic or orderly behavior of the iterated map $F^{n}(\cdot)$.

Let $f(x, v)$ be the same as defined in (2.13). For each $F_{i}, i=1,2,3$, define $g_{i}$ through

$$
u_{i}=F_{i}(v)=v+g_{i}(v), \quad i=1,2,3
$$

where

$$
\left.\begin{array}{l}
g_{i}(v)=\sqrt[3]{-\frac{v}{\beta}+\sqrt{\mathcal{D}}}+\sqrt[3]{-\frac{v}{\beta}-\sqrt{\mathcal{D}}}, \quad i=1,3, \\
g_{2}(v)=\bar{\omega} \sqrt[3]{-\frac{v}{\beta}+\sqrt{\mathcal{D}}}+\omega \sqrt[3]{-\frac{v}{\beta}-\sqrt{\mathcal{D}}}, \\
\operatorname{Dom}\left(g_{1}\right)=\left\{v \mid v<-v^{*}\right\}, \operatorname{Dom}\left(g_{2}\right)=\left\{v \mid-v^{*}<v<v^{*}\right\}, \\
\operatorname{Dom}\left(g_{3}\right)=\left\{v \mid v>v^{*}\right\} .
\end{array}\right\}
$$

(From now on, we will also let $g_{2}$ and $g_{3}$ be defined at $-v^{*}$ and $v^{*}$, respectively, through continuity.) Similarly to (3.3), we easily see that

$$
f\left(g_{i}(v), v\right)=0, \quad i=1,2,3, \text { for } v \in \text { Domain of } g_{i} .
$$

At $v=v^{*}$, we have $\mathcal{D}=0$, cf. (2.19), and, thus, $f\left(x, v^{*}\right)=0$ has a double root; see (2.8). We have

$$
f\left(x, v^{*}\right)=\beta\left(x+2 x^{*}\right)\left(x-x^{*}\right)^{2}=0,
$$

where $x^{*}$ is given in $(2.15)$. Since $f\left(g_{i}\left(v^{*}\right), v^{*}\right)=0$ for $i=2,3$, we get

$$
\left\{\begin{array}{l}
g_{2}\left(v^{*}\right)=x^{*} \\
g_{3}\left(v^{*}\right)=-2 x^{*}
\end{array}\right.
$$

where $g_{3}$ has been extended and defined at $v^{*}$ through right continuity. From (3.23), we get

$$
f(x, v *) \begin{cases}>0, & \text { if } x>x^{*} \text { or }-2 x^{*}<x<x^{*}, \\ =0, & \text { if } x=x^{*} \text { or } x=-2 x^{*}, \\ <0, & \text { if } x<-2 x^{*} .\end{cases}
$$

We list a few properties of the multivalued function $F$ in the following.

(Q.1) The function $g_{2}(v)$ is strictly increasing for $v \in\left(-v^{*}, v^{*}\right)$. Consequently, $u=F_{2}(v)=v+g_{2}(v)$ satisfies $F_{2}^{\prime}(v)>1$ for all $v \in\left(-v^{*}, v^{*}\right)$. 
Let $v>v^{*}$ so $v$ belongs to the domain of definition of $g_{3}$. Since $g_{3}\left(v^{*}\right)=-2 x^{*}$ by (3.24) we have

$$
f\left(g_{3}(v), v^{*}\right)=f\left(g_{3}(v), v\right)-2\left(v-v^{*}\right)=-2\left(v-v^{*}\right)<0 .
$$

Therefore by the third inequality in $(3.25)$, we get $g_{3}(v)<-2 x^{*}$. We now define a function $a(v)$ of $v$ through

$$
g_{3}(v)=\left\{\begin{array}{l}
-a(v) x^{*}, \text { where } a(v)>2, \text { for } v>v^{*}, \\
-a\left(v^{*}\right) x^{*}=-2 x^{*}, \text { for } v=v^{*} .
\end{array}\right.
$$

Lemma 3.1. Let $\alpha>1$ and be fixed. For $v \geq v^{*}, a(v)$ satisfies $a(v) \geq 2$ and

$$
[a(v)-2][a(v)+1]^{2}=\frac{6}{\alpha-1} \frac{v-v^{*}}{x^{*}} .
$$

Furthermore, $a:\left[v^{*}, \infty\right) \rightarrow[2, \infty)$ is strictly increasing and onto, and

$$
F_{3}^{\prime}(v)=1-\frac{2}{\alpha-1} \frac{1}{\left[a(v)^{2}-1\right]} .
$$

Proof. Since

$$
f\left(g_{3}(v), v\right)=f\left(-a(v) x^{*}, v\right)=0,
$$

we have

$$
-\beta a(v)^{3} x^{* 3}-(1-\alpha) a(v) x^{*}+2 v=0 .
$$

Also

$$
\begin{aligned}
& f\left(-2 x^{*}, v^{*}\right)=0, \\
& -8 \beta x^{* 3}-2(1-\alpha) x^{*}+2 v^{*}=0 .
\end{aligned}
$$

Subtracting (3.29) from (3.30), we get

$$
\begin{aligned}
& \beta x^{* 3}\left[a(v)^{3}-8\right]+(1-\alpha)[a(v)-2] x^{*}=2\left(v-v^{*}\right), \\
& \beta x^{* 2}[a(v)-2]\left[a(v)^{2}+2 a(v)+4\right]+(1-\alpha)[a(v)-2]=\frac{2\left(v-v^{*}\right)}{x^{*}} .
\end{aligned}
$$

From (2.15), we have

$$
x^{* 2}=\frac{\alpha-1}{3 \beta} .
$$

Hence

$$
\begin{aligned}
& {[a(v)-2]\left\{\beta \cdot\left(\frac{\alpha-1}{3 \beta}\right)\left[a(v)^{2}+2 a(v)+4\right]-(\alpha-1)\right\}=\frac{2\left(v-v^{*}\right)}{x^{*}},} \\
& {[a(v)-2](\alpha-1) \cdot\left[a(v)^{2}+2 a(v)+4-3\right]=\frac{6\left(v-v^{*}\right)}{x^{*}},}
\end{aligned}
$$

which is (3.27).

The rest of the proof can be done by differentiating (3.27) with respect to $\alpha$. We omit the details.

(Q.2) Let $\alpha>1$. Then for

$$
g_{3}\left(v^{*}+x^{*}\right)=-a\left(v^{*}+x^{*}\right) \cdot x^{*} \equiv-\tilde{a} x^{*},
$$


we have

$$
\tilde{a}>2, \quad(\tilde{a}-2)(\tilde{a}+1)^{2}=\frac{6}{\alpha-1} .
$$

(Q.3) If $\alpha>5 / 3$, then $0<F_{3}^{\prime}(v)<1$ for all $v \geq v^{*}$. By symmetry, for $v<-v^{*}$, we have $0<F_{1}^{\prime}(v)<1$.

Lemma 3.2. Let $\alpha>1$. For $v \in\left[-v^{*} \cdot v^{*}\right]$, define $g_{2}(v)=b(v) x^{*}$. Then

$$
\begin{gathered}
b(-v)=-b(v), \quad-1 \leq b(v) \leq 1, \\
{[b(v)-1]^{2}[b(v)+2]=-\frac{6}{\alpha-1} \frac{v-v^{*}}{x^{*}}, \quad v \in\left[0, v^{*}\right] .}
\end{gathered}
$$

If $v \in\left[0, v^{*}\right]$, then $0 \leq b(v) \leq 1$. Furthermore,

$$
F_{2}^{\prime}(v)=1+\frac{2}{(\alpha-1)\left[1-b(v)^{2}\right]}, \quad \text { for } \quad v \in\left[0, v^{*}\right] .
$$

Proof. The oddness of $b: b(-v)=-b(v)$, is obvious. Because

$$
0 \leq g_{2}(v) \leq x^{*}, \quad \text { for } \quad v \in\left[0, v^{*}\right]
$$

from the definition of $b(v)$, we get (3.32). We have

$$
0=f\left(g_{2}(v), v\right)=f\left(b(v) x^{*}, v\right)=0,
$$

so

$$
\beta b^{3} x^{* 3}+(1-\alpha) b x^{*}+2 v=0, \quad b \equiv b(v) .
$$

Also,

$$
\begin{aligned}
& f\left(-2 x^{*}, v^{*}\right) \\
& \quad=-8 \beta x^{* 3}-2(1-\alpha) x^{*}+2 v^{*}=0 \quad \text { from (3.30). }
\end{aligned}
$$

Subtracting (3.30) from (3.35), and using (3.31) we get (3.34).

To obtain $F_{2}^{\prime}(v)$, we differentiate $b(v)$ in $(3.33)$ and use $F_{2}^{\prime}(v)=1+b^{\prime}(v) x^{*}$. The work is straightforward, and we obtain (3.34).

Remark 3.1. Lemma 3.2 consists of dimensionless statements. It looks surprising at first that $a(v)$ and $b(v)$ therein depend only on $\alpha$ but not on $\beta$. But after checking through their proofs, it is easy for one to discover that $\beta$ plays the role of a scaling factor.

(Q.4) Let $\alpha>1$. Assume that

$$
v^{*}-2 x^{*}>0 .
$$

Define $\tilde{b} \in \mathbf{R}$ by

$$
g_{2}\left(v^{*}-2 x^{*}\right)=\tilde{b} x^{*} .
$$

Then $0<\tilde{b}<1$, and $\tilde{b}$ satisfies

$$
(\tilde{b}+2)(\tilde{b}-1)^{2}=\frac{12}{\alpha-1} .
$$


Lemma 3.3 (Overlapping condition). Let $\alpha>\frac{11}{8}$. Then we have

$$
F_{3}\left(F_{2}\left(v^{*}\right)\right)>F_{2}\left(F_{3}\left(v^{*}\right)\right) \text {, }
$$

where each $F_{i}$, first defined as in (3.21), has been extended by continuity.

Proof. By (3.20) and (3.24), we have

$$
\begin{aligned}
& F_{2}\left(v^{*}\right)=v^{*}+x^{*}, \\
& F_{3}\left(F_{2}\left(v^{*}\right)\right)=F_{3}\left(v^{*}+x^{*}\right)=\left(v^{*}+x^{*}\right)+g_{3}\left(v^{*}+x^{*}\right)=\left(v^{*}+x^{*}\right)-\tilde{a} x^{*} .
\end{aligned}
$$

On the other hand, from (3.20) and (3.24), we also have

$$
\begin{aligned}
& F_{3}\left(v^{*}\right)=v^{*}-2 x^{*}, \\
& F_{2}\left(F_{3}\left(v^{*}\right)\right)=F_{2}\left(v^{*}-2 x^{*}\right)=\left(v^{*}-2 x^{*}\right)+\tilde{b} x^{*}, \text { cf. (Q.4). }
\end{aligned}
$$

Comparing between (3.40) and (3.41), we see that (3.39) holds iff

$$
\left(v^{*}+x^{*}\right)-\tilde{a} x^{*}>\left(v^{*}-2 x^{*}\right)+\tilde{b} x^{*},
$$

i.e., iff

$$
3-\tilde{a}>\tilde{b}
$$

By (Q.2) and (Q.4), we have

$$
\begin{aligned}
& (\tilde{a}-2)(\tilde{a}+1)^{2}=\frac{6}{\alpha-1}, \\
& (\tilde{b}+2)(\tilde{b}-1)^{2}=\frac{12}{\alpha-1} .
\end{aligned}
$$

Since the function $G(a) \equiv(a-2)(a+1)^{2}$ is strictly increasing for $a>2$, if we can show that

$$
a_{0} \equiv 3-\tilde{b} \quad \text { satisfies } \quad\left(a_{0}-2\right)\left(a_{0}+1\right)^{2}>\frac{6}{\alpha-1},
$$

then by the strict increasing property of $G$, we will have

$$
a_{0}=3-\tilde{b}>\tilde{a},
$$

proving (3.42). But the verification of (3.44) is straightforward.

The term "overlapping" comes from Keener [11, p. 590]. Therefore in our paper, we will be dealing primarily with the overlapping case (while [11] seems to be more concerned with the "nonoverlapping" case).

\section{Chaotic or Nonchaotic Vibrations as Determined By $\boldsymbol{\alpha}$}

Separate mathematical treatments are required for parameter $\alpha$ in various numerical ranges. Some prerequisites for this section are given in Appendix A, which should be consulted first.

The important numerical ranges for $\alpha$ can be given as follows:

$$
\left.\begin{array}{ll}
F_{3}\left(v^{*}\right)=v^{*}-2 x^{*}>0 & \Leftrightarrow \alpha>7 ; \\
F_{3}\left(v^{*}+x^{*}\right)>0 & \Leftrightarrow \alpha>4.5103 ; \\
F_{3}\left(v^{*}\right)=v^{*}-2 x^{*}<-v^{*} & \Leftrightarrow \alpha<4 ; \\
F_{3}\left(v^{*}+x^{*}\right)>-v^{*} & \Leftrightarrow \alpha>2.9312 .
\end{array}\right\}
$$

These values of $\alpha$ can all be computed based upon Lemma 3.1. 
Let us define preimages $v_{i}^{*}$ of $v^{*}$ by

$$
v_{0}^{*} \equiv v^{*}, \quad F\left(v_{i+1}^{*}\right)=v_{i}^{*}, \quad v_{i+1}^{*}<v_{i}^{*}, \quad i=0,1,2, \ldots .
$$

Each $v_{i}^{*}$ depends on $\alpha$ : $v_{i}^{*}=v_{i}^{*}(\alpha)$. We will omit this $\alpha$ dependence unless clarity is required. From $(3.20),(3.21)$ and Lemma 3.2 , each $v_{j}^{*}$ is recursively representable as

$$
v_{j}^{*}=v_{j-1}^{*}-b_{j} x^{*}=\left(v_{j-2}^{*}-b_{j-1} x^{*}\right)-b_{j} x^{*}=\cdots=v^{*}-\left(\sum_{i=1}^{j} b_{i}\right) x^{*},
$$

where each $b_{j}$ is the unique real solution of

$$
\left(b_{j}-1\right)^{2}\left(b_{j}+2\right)=\frac{6}{\alpha-1} \sum_{i=1}^{j} b_{i}, \quad 0 \leq b_{j} \leq 1, j=1,2, \ldots .
$$

Our justification of the results in Table 1 is based primarily on the properties of $F$ given in (4.1). We therefore divide the discussion according to the numerical regimes of $\alpha$ in the following subsections.

4.1. $\boldsymbol{\alpha} \geq 7$. In this case $u=F(v)$ has (besides the unique fixed point 0 ) two invariant (rectangular) regions in the $(u, v)$-plane:

$$
R_{1}=I \times I, \quad R_{2}=(-I) \times(-I), \quad I \equiv\left[v^{*}-2 x^{*}, v^{*}+x^{*}\right] .
$$

$R_{1}$ and $R_{2}$ are disconnected if $\alpha>7$, and connected at the origin if $\alpha=7$. Any iterate $u_{n}=F^{n}\left(v_{0}\right), v_{0} \neq 0$, will eventually be trapped into $R_{1}$ or $R_{2}$.

Let us define $n_{1}(\alpha)$ to be a positive integer s.t.

$$
v^{*}-2 x^{*} \leq v_{n_{1}(\alpha)}^{*}<v_{n_{1}(\alpha)-1}^{*}<\cdots<v_{1}^{*}<v_{0}^{*}=v^{*} .
$$

Also, define $I_{j}=\left[v_{j}^{*+}, v_{j-1}^{*-}\right], j=1,2, \ldots, n_{1}(\alpha)$. Using (4.2) it is not too hard to compute that

$$
\begin{aligned}
& n_{1}(\alpha) \geq 3, \text { if } \quad 7 \leq \alpha \leq 13.7853 ; \\
& 3>n_{1}(\alpha) \geq 2, \quad \text { if } \quad \alpha>13.7853 .
\end{aligned}
$$

Theorem 4.1. Let $7 \leq \alpha \leq 13.7853$. Then we have a shift sequence

$$
I_{n_{1}(\alpha)} \longrightarrow I_{n_{1}(\alpha)-1} \longrightarrow \cdots \longrightarrow I_{1} \longrightarrow I_{n_{1}(\alpha)} \cup I_{n_{1}(\alpha)-1}
$$

Consequently, $\rho_{I_{n_{1}(\alpha)}} \supseteq\left(0, \frac{1}{n_{1}(\alpha)}\right]$, and the mapping $u=F(v)$ is chaotic in $R_{1}$.

Proof. For $7 \leq \alpha \leq 13.7853$, we have (4.5). Further, one can use Lemma 3.1 and (4.2) to show that $F\left(v^{*}+x^{*}\right) \geq v_{n_{1}(\alpha)-2}^{*}$. Therefore the proof follows by an application of Theorem A.1 in the Appendix.

To prove that there is chaos for $\alpha>13.7853$, direct constructions of shift sequences become difficult. We need to resort to more sophisticated machinery developed by M.I. Malkin [16, 17]. In the rest of this subsection, we will show that our map $F$ satisfies the requisite properties in Appendix B.

The hyperbolicity of a map is well known to be playing a crucial role in causing chaos. Here we first establish the (strictly expansive) hyperbolicity of $F^{2}$ in the following.

Lemma 4.1. Let $v \neq v^{*}, v \in\left[v^{*}-2 x^{*}, v^{*}+x^{*}\right](c f .(4.3))$. Then $\left(F^{2}\right)^{\prime}(v)>q>1$ for some $q>1$ for all $\alpha \geq 7$. 
Proof. First, we claim that $u=F(v)$ satisfies $F^{\prime \prime}(v)>0$ for $v \in\left[v^{*}-2 x^{*}, v^{*}+x^{*}\right]$, $v \neq v^{*}$. Write

$$
u=F(v)=\left\{\begin{array}{ll}
v+g_{2}(v), & v \in\left[v^{*}-2 x^{*}, x^{*}\right), \\
v+g_{3}(v), & v \in\left(v^{*}, v^{*}+x^{*}\right),
\end{array} \quad\right. \text { cf. (3.20), (3.21). }
$$

Then from (2.15) and (3.4),

$$
g_{i}^{\prime}(v)=\frac{-2}{3 \beta\left(\left[g_{i}(v)\right]^{2}-x^{* 2}\right)}>0, \quad i=2,3,
$$

because

$$
g_{3}(v)<-2 x^{*} \text { for } v>v^{*} \text {, and } 0 \leq g_{2}(v) \leq x^{*} \text { for } v \in\left[0, v^{*}\right] .
$$

Differentiating (4.7) again and using (4.8), we get

$$
g_{i}^{\prime \prime}(v)=\frac{-2 g_{i}(v)\left[g_{i}^{\prime}(v)\right]^{2}}{g_{i}(v)^{2}-x^{* 2}}>0, \quad i=2,3
$$

Hence

$$
F^{\prime \prime}(v)=g_{i}^{\prime \prime}(v)>0 \text { for } i=2,3 .
$$

Now for $v \in\left(v_{1}^{*}, v^{*}\right)$ or $v \in\left(v^{*}, v^{*}+x^{*}\right)$,

$$
\begin{aligned}
{[F(F(v))]^{\prime} } & =F^{\prime}(F(v)) F^{\prime}(v)>F^{\prime}\left(v^{*}-2 x^{*}\right) \cdot F^{\prime}\left(v^{*}+\right), \text { by }(4.9) ; \\
F^{\prime}\left(v^{*}-2 x^{*}\right) & =1+g_{2}^{\prime}\left(v^{*}-2 x^{*}\right) \\
& =1-\frac{2}{3 \beta\left[g_{2}\left(v^{*}-2 x^{*}\right)^{2}-x^{* 2}\right]} \\
F^{\prime}\left(v^{*}+\right) & =1-\frac{2}{3 \beta\left[g_{3}\left(v^{*}\right)^{2}-x^{* 2}\right]} \\
& =1-\frac{2}{3 \beta} \frac{1}{4\left(x^{*}\right)^{2}-x^{* 2}}=1-\frac{2}{3} \frac{1}{\alpha-1} ;(\text { by }(3.24))
\end{aligned}
$$

To determine $g_{2}\left(v^{*}-2 x^{*}\right)$ in (4.11), we use (3.33):

$$
g_{2}\left(v^{*}-2 x^{*}\right)=b x^{*}, \quad 0<b<1, \quad(b+2)(b-1)^{2}=-\frac{12}{\alpha-1} .
$$

Using (4.9)-(4.13), we get

$$
F^{\prime}\left(v^{*}-2 x^{*}\right) F^{\prime}\left(v^{*}+\right)>\left[1-\frac{2}{\left(b^{2}-1\right)(\alpha-1)}\right]\left[1-\frac{2}{3} \frac{1}{\alpha-1}\right]>1
$$

if and only if

$$
-2+\frac{2}{\alpha-1}<b^{2}
$$

If $\alpha \geq 7$, then

$$
-2+\frac{2}{\alpha-1}<0 \leq b^{2}
$$

and therefore from (4.10) and (4.14), $\left(F^{2}\right)^{\prime}(v)>q>1$ for some $q$ for all $\alpha \geq 7$ and for all $v$ in the specified range.

Lemma 4.2. Let $I=\left[v^{*}-2 x^{*}, v^{*}+x^{*}\right]$ as in (4.3) and let $\alpha \geq 7$. Then $F \in \mathcal{F}_{L}(I)$ (cf. Appendix B) and $h(F)>0$, where $h(F)$ is the topological entropy of $F$ on $I$ as defined in (B.1). 
Proof. For each $n \in \mathbb{Z}^{+}$, write

$$
d_{0} \equiv v^{*}-2 x^{*}<d_{1}<d_{2}<\cdots<d_{m_{n}}<d_{m_{n}+1} \equiv v^{*}+x^{*},
$$

where $d_{1}, d_{2}, \ldots, d_{m_{n}}$ are the points of discontinuities of $F^{2 n}$. By Lemma 4.1, $\left(F^{2 n}\right)^{\prime}(v)>q^{n}>1$ for all $v \in\left[v^{*}-2 x^{*}, v^{*}+x^{*}\right], v \neq d_{i}, i=1, \ldots, m_{n}$. Because $I$ is invariant under $F^{2 n}$, we have

$$
3 x^{*}=|I|>\left|F^{2 n}\left(d_{j}, d_{j+1}\right)\right|>q^{n}\left|\left(d_{j}, d_{j+1}\right)\right|, \quad j=0, \ldots, m_{n},
$$

where the absolute value bars denote the measure of the set. Thus

$$
\begin{aligned}
& \left|\left(d_{j}, d_{j+1}\right)\right|<3 x^{*} \cdot q^{-n}, \\
& 3 x^{*}=\sum_{j=0}^{m_{n}}\left|\left(d_{j}, d_{j+1}\right)\right| \leq 3 x^{*} \sum_{j} q^{-n}=3 x^{*} \cdot\left(m_{n}+1\right) q^{-n}, \\
& m_{n} \geq q^{n}-1 .
\end{aligned}
$$

Therefore

$$
h(F)=\lim _{n \rightarrow \infty} \frac{\log m_{n}}{n} \geq \lim _{n \rightarrow \infty} \frac{\log \left(q^{n}-1\right)}{2 n}=\frac{\ln q}{2}>0 .
$$

Theorem 4.2. For $\alpha \geq 13.7853$, there exists $k \in \mathbb{Z}^{+}$such that $F^{k}$ is chaotic (in the renormalized sense of Keener according to Corollary B.1).

Proof. We wish to apply Corollary B.1 to $F$ on $I=\left[v^{*}-2 x^{*}, v^{*}+x^{*}\right]$. First, we note from Lemma 3.3 that $F\left(v^{*}-2 x^{*}\right)<F\left(v^{*}+x^{*}\right)$. In view of Lemma 4.2, we need only verify that $\left(\mathrm{F}-\mathrm{II}^{\prime}\right)$ in Appendix B is satisfied, i.e., $\mathcal{D} \equiv \bigcup_{n=0}^{\infty} F^{-n}\left(v^{*}\right)$ is dense in $\left[v^{*}-2 x^{*}, v^{*}+x^{*}\right]$. Let $J$ be any open subinterval of $I$. Then from Lemma 4.1, $\left|F^{2 k}(J)\right|>q^{k}|J|$ for some $q>1$, provided that none of $J, F^{2}(J), \ldots,\left(F^{2}\right)^{k-1}$ contains $v^{*}$. But $\left|F^{2 k}(J)\right|<|I|$, so for $k$ sufficiently large, there will be a contradiction. Hence there exists some $k_{0} \in \mathbb{Z}^{+}$s.t. $F^{2 k_{0}}(J)$ contains $v^{*}$, i.e., $F^{-2 k_{0}}\left(v^{*}\right) \in J$. Therefore $\mathcal{D}$ is dense in $I$.

Note that Theorem 4.2 is also valid for $\alpha \geq 7$ and thus its proof applies to Theorem 4.1 as well. But the senses of chaos in Theorems 4.1 and 4.2 are slightly different. We also note that we have not been able to overcome the restriction of uniform boundedness of derivative made by Lasota and Yorke in [12]. Otherwise we could have applied the results in [12] to obtain chaos under the assumptions of Theorem 4.2.

4.2. 4.5103 $<\boldsymbol{\alpha}<7$. For $\alpha$ in this range, a trapping region of $F$ is the square

$$
R=\left[-\left(v^{*}+x^{*}\right), v^{*}+x^{*}\right] \times\left[-\left(v^{*}+x^{*}\right), v^{*}+x^{*}\right],
$$

where $F$ has two jump discontinuities, at $v= \pm v^{*}$.

Theorem 4.3. Let $\alpha \in(4.5103,7)$ and $\beta>0$. Then

$$
\text { range } \rho_{I_{0}} \supseteq(0, \delta] \text {, for some } \delta>0 \text { for some closed interval } I_{0} \text {. }
$$

Consequently, $F$ is chaotic with a single strange attractor $R$ given by (4.16). 
Proof. The arguments are a simple adaptation of Keener's [11, Lemma 4.1]. Since $F$ is continuous on $\left[v^{*}, v^{*}+x^{*}\right]$, between $F\left(v^{*}\right)<0$ and $F\left(v^{*}+x^{*}\right)>0$ there exists a $\theta \in\left(v^{*}, v^{*}+x^{*}\right)$ s.t. $F(\theta)=0$. Let $k$ be a positive integer s.t. $v_{k}^{*} \leq F\left(-v^{*-}\right)<v_{k-1}^{*}$. Define

$$
\begin{aligned}
I_{0} & =\left[v^{*}, v^{*}+x^{*}\right], I_{1}=\left[v_{k+2}^{*+}, v_{k+1}^{*-}\right], I_{2}=\left[v_{k+1}^{*+}, v_{k}^{*-}\right], \ldots, I_{k+2}=\left[v_{1}^{*+}, v_{0}^{*-}\right], \\
I_{k+3} & =\left[v_{0}^{*+}, \theta^{-}\right], I_{k+4}=-I_{2}, I_{k+3}=-I_{3}, \ldots, I_{2 k+4}=-I_{k+2}, \\
I_{2 k+5} & =-I_{k+3} .
\end{aligned}
$$

Then we have a shift sequence

$$
I_{1} \longrightarrow I_{2} \longrightarrow \cdots \longrightarrow I_{k+2} \longrightarrow I_{k+3} \longrightarrow \cdots \longrightarrow I_{2 k+5} \longrightarrow I_{1} \cup I_{2} .
$$

By Keener [11, Corollary 4.2], we have

$$
\text { range } \rho_{I_{0}} \supseteq\left[0, \frac{1}{k+1}\right] \text {, }
$$

where $0 \in$ range $\rho_{I_{0}}$ because $F(0)=0$. The proof follows.

4.3. $1<\boldsymbol{\alpha} \leq$ 4.5103. Define $n_{2}(\alpha)$ to be the nonnegative integer satisfying

$$
v_{n_{2}(\alpha)+1}^{*}<-F_{3}\left(v^{*}+x^{*}\right)<v_{n_{2}(\alpha)}^{*}<\cdots<v_{1}^{*}<v^{*} .
$$

Computing from (4.2), we obtain the following values:

$$
\left.\begin{array}{ll}
n_{2}(\alpha)=4: & 4.1415<\alpha<4.5103 \\
n_{2}(\alpha)=3: & 3.9759<\alpha<4.1415 \\
n_{2}(\alpha)=2: & 3.7444<\alpha<3.9759 \\
n_{2}(\alpha)=1: & 3.4172<\alpha<3.7444 \\
n_{2}(\alpha)=0: & 2.9312<\alpha<3.4172
\end{array}\right\}
$$

We will classify the asymptotic behavior of $F^{n}$ according to (4.18). For brevity, we just write $n(\alpha)$ for $n_{2}(\alpha)$.

Theorem 4.4. Let $\alpha \in(3.7444,4.5103]$ so that $n(\alpha) \geq 2$. Then the map $F$ is chaotic.

Proof. First consider $n(\alpha) \geq 3$. Define $\eta$ (to be the unique number s.t.)

$$
\begin{aligned}
& \text { (i) } \eta=v^{*} \text { if } \alpha \geq 4 ; \\
& \text { (ii) } \eta \in\left(v^{*}, v^{*}+x^{*}\right), \quad F_{3}(\eta)=-v^{*} \text {, if } \alpha<4 .
\end{aligned}
$$

We can construct the following shift sequence

$$
\begin{aligned}
I_{1} & =\left[v_{n(\alpha)}^{*+}, v_{n(\alpha)-1}^{*-}\right] \longrightarrow I_{2}=\left[v_{n(\alpha)-1}^{*+}, v_{n(\alpha)-2}^{*-}\right] \longrightarrow \cdots \longrightarrow I_{n(\alpha)}=\left[v_{1}^{*+}, v^{*-}\right] \\
& \longrightarrow I_{n(\alpha)+1}=\left[\eta^{+},\left(v^{*}+x^{*}\right)^{-}\right] \longrightarrow I_{n(\alpha)+2}=\left[\left(-v^{*}\right)^{+},\left(-v_{1}^{*}\right)^{-}\right] \\
& \longrightarrow I_{n(\alpha)+3} \\
& =\left[\left(-v^{*}-x^{*}\right)^{+},(-\eta)^{-}\right] \longrightarrow I_{1} \cup I_{2} .
\end{aligned}
$$

If $n_{2}(\alpha)=2$, we let $\eta$ satisfy (4.19). Further, define $\varphi^{i}, i=1, \ldots, 6$, s.t.

$$
\begin{array}{ll}
F_{1}\left(\varphi^{1}\right)=v_{2}^{*}, \varphi^{1} \in\left(-v^{*}-x^{*},-v^{*}\right) ; & F_{3}\left(\varphi^{4}\right)=\varphi^{3}, \varphi^{4} \in\left(\eta, v^{*}+x^{*}\right) ; \\
F_{2}\left(\varphi^{2}\right)=\varphi^{1}, \varphi^{2} \in\left(-v^{*},-v_{1}^{*}\right) ; & F_{2}\left(\varphi^{5}\right)=\varphi^{4}, \varphi^{5} \in\left(v_{1}^{*}, v^{*}\right) ; \\
F_{2}\left(\varphi^{3}\right)=\varphi^{2}, \varphi^{3} \in\left(-v_{1}^{*},-v_{2}^{*}\right) ; & F_{2}\left(\varphi^{6}\right)=\varphi^{5}, \varphi^{6} \in\left(v_{2}^{*}, v_{1}^{*}\right) .
\end{array}
$$


Then we can construct a shift sequence

$$
\begin{aligned}
I_{1}= & {\left[v_{2}^{*+}, \varphi^{6-}\right] \longrightarrow I_{2}=\left[v_{1}^{*+}, \varphi^{5-}\right] \longrightarrow I_{3}=\left[\eta^{+}, \varphi^{4-}\right] } \\
& \longrightarrow I_{4}=\left[\left(-v^{*}\right)^{+},\left(-v_{1}^{*}\right)^{-}\right] \longrightarrow I_{5}=\left[\left(-v^{*}-x^{*}\right)^{+},(-\eta)^{-}\right] \longrightarrow I_{1} \cup I_{2} .
\end{aligned}
$$

The proof is complete.

Remark 4.1. Theorem 4.4 shows that for $\alpha \in(3.7444,4.5103]$, the system is chaotic, i.e., the range of the rotation number contains a subinterval. However, there is a qualitative difference between the cases of $\alpha>4$ and $\alpha<4$. For $\alpha>4$, numerical experiments show that there is a single strange attractor. For $\alpha<4$, numerical simulation shows that the iterates converge to a unique period- 2 orbit (i.e., the infinitely many other periodic points become invisible to the computer). In what follows, we will show that for $\alpha<4$, there is a unique period-2 orbit attracting almost all points. (Thus the occurrence of chaos when $\alpha<4$ is weak, termed "transient chaos" in Moon [18].)

Theorem 4.5. Let $\alpha \in(2.9312,4)$. Then there exists a unique attracting period-2 point $p \in\left[v^{*}, \eta\right], F^{2}(p)=p$, where $\eta$ is the unique number in $\left(v^{*}, v^{*}+x^{*}\right)$ satisfying $F_{3}(\eta)=-v^{*}$.

Proof. Note from (4.1) that $F_{3}\left(v^{*}\right)<-v^{*}$ and $F_{3}\left(v^{*}+x^{*}\right)>-v^{*}$, and so $\eta \in$ $\left(v^{*}, v^{*}+x^{*}\right)$ uniquely exists. Since $F_{3}(\eta)=-v^{*}$ and $0<F_{3}^{\prime}(v)<1$ (by (Q.3)) on $\left[v^{*}, \eta\right]$, we have $F_{3}\left(\left[v^{*}, \eta\right]\right) \subseteq\left[-\eta,-v^{*}\right]$.

Similarly, $F_{1}\left(\left[-\eta,-v^{*}\right]\right) \subseteq\left[v^{*}, \eta\right]$, and $0<F_{1}^{\prime}(v)<1$ on $\left[-\eta,-v^{*}\right]$.

Therefore $F_{1} \circ F_{3}:\left[v^{*}, \eta\right] \rightarrow\left[v^{*}, \eta\right]$ is a contraction, and hence a unique 2-periodic point $p$ exists in $\left[v^{*}, \eta\right]$, which is attracting.

Let us further classify the behavior into the following subcases.

Case (i) $n(\alpha)=0,2.9312<\alpha<3.4172$

In this case, we have not only an attracting period- 2 orbit, but also some period- 4 orbit.

Let $\eta$ be the same as in Lemma 4.1. Let $\eta_{1} \in\left(-v^{*}, v^{*}\right)$ satisfy $F_{2}\left(\eta_{1}\right)=\eta$. Numerical computation indicates that for all $\alpha$ : $2.9312<\alpha<3.4172$, we have $F_{1}\left(-v^{*}-x^{*}\right)<\eta_{1}$. Let $p_{1} \in\left[v^{*}, \eta\right]$ be the period-2 point $p$ as promised in Theorem 4.5 , and let $p_{2}=F_{3}\left(p_{1}\right)$. The stable set $W^{s}\left(p_{1}, p_{2}\right)$ is the domain of attraction of the period- 2 orbit $\left\{p_{1}, p_{2}\right\}$. Thus

$$
F_{2}\left(\left[F_{1}\left(-v^{*}-x^{*}\right), \eta_{1}\right]\right) \subseteq\left[v^{*}, \eta\right] \text {, implying }\left[F_{1}\left(-v^{*}-x^{*}\right), \eta_{1}\right] \subseteq W^{s}\left(p_{1}, p_{2}\right) .
$$

Also

$$
\left[-\eta,-v^{*}\right],\left[-\eta_{1}, F_{3}\left(v^{*}+x^{*}\right)\right] \subseteq W^{s}\left(p_{1}, p_{2}\right) .
$$

Let $\left[\xi_{1}, v^{*}+x^{*}\right] \equiv F_{3}^{-1}\left(\left[-\eta_{1}, F_{3}\left(v^{*}+x^{*}\right)\right]\right)$. Then $\left[\xi_{1}, v^{*}+x^{*}\right] \subseteq W^{s}\left(p_{1}, p_{2}\right)$.

Let $F_{2}\left(b_{1}\right)=\xi_{1}$. Then $\left[b_{1}, v^{*}\right] \subseteq W^{s}\left(p_{1}, p_{2}\right)$, as well as $\left[-v^{*},-b_{1}\right] \subseteq W^{s}\left(p_{1}, p_{2}\right)$. Define $\delta_{1}$ by $\left[\eta, \delta_{1}\right]=F_{3}^{-1}\left(\left[-v^{*},-b_{1}\right]\right)$. Then $\left[\eta, \delta_{1}\right] \subseteq W^{s}\left(p_{1}, p_{2}\right)$. Define $a_{1}=$ $F_{2}^{-1}\left(\delta_{1}\right)$. Then $\left[\eta_{1}, a_{1}\right],\left[-a_{1},-\eta_{1}\right] \subseteq W^{s}\left(p_{1}, p_{2}\right)$.

Define $\xi_{2}=F_{3}^{-1}\left(-a_{1}\right)$. Then $\left[\xi_{2}, \xi_{1}\right] \subseteq W^{s}\left(p_{1}, p_{2}\right)$.

Continuing this process, we obtain two sequences, $\delta_{n}, \xi_{n}$ s.t.

$$
\begin{gathered}
\delta_{1}<\delta_{2}<\cdots<\delta_{n}<\cdots<\xi_{n}<\cdots<\xi_{2}<\xi_{1}, \\
{\left[v^{*}, \delta_{n}\right],\left[\xi_{n}, v^{*}+x^{*}\right] \subseteq W^{s}\left(p_{1}, p_{2}\right), \quad n=1,2, \ldots .}
\end{gathered}
$$


Extensive numerical experiments indicate that the period-2 orbit $\left\{p_{1}, p_{2}\right\}$ attracts every point (except the origin). Therefore we conjecture that $\hat{\delta} \equiv \lim _{n \rightarrow \infty} \delta_{n}, \hat{\xi} \equiv$ $\lim _{n \rightarrow \infty} \xi_{n}$, satisfy $\hat{\delta}=\hat{\xi}$, and $\hat{\delta}$ has period four.

But if $\hat{\delta}<\hat{\xi}$, then we have at least two points $\hat{\delta}$ and $\hat{\xi}$, with period four and two distinct period- 4 orbits.

Case (ii) $n(\alpha)=1,3.4172<\alpha<3.7444$

Let $\eta_{1}=F_{2}^{-1}(\eta)$ and $\eta_{2}=F_{2}^{-1}\left(\eta_{1}\right)$. First, we consider

$$
F_{1}\left(-v^{*}-x^{*}\right)<\eta_{2}<v_{1}^{*}<\eta_{1}<v^{*}<\eta \text {, i.e., } 3.5746<\alpha<3.7444 .
$$

Then similarly as in Case (i),

$$
\begin{array}{r}
{\left[v^{*}, \eta\right],\left[-\eta,-v^{*}\right],\left[v_{1}^{*}, \eta_{1}\right],\left[-\eta_{1},-v_{1}^{*}\right],\left[F_{1}\left(-v^{*}-x^{*}\right), \eta_{2}\right],} \\
{\left[-\eta_{2}, F_{3}\left(v^{*}+x^{*}\right)\right] \subseteq W^{s}\left(p_{1}, p_{2}\right) .}
\end{array}
$$

Let $A_{0}=F_{3}^{-1}\left(\left[-\eta_{1},-v_{1}^{*}\right]\right), A_{1}=F_{3}^{-1}\left(\left[-\eta_{2}, F_{3}\left(v^{*}+x^{*}\right)\right]\right)$. Then $A_{0}, A_{1} \subseteq W^{s}\left(p_{1}, p_{2}\right)$. Next, let $A_{i}^{1}=F_{2}^{-1}\left(A_{i}\right), A_{i}^{2}=F_{2}^{-1}\left(A_{i}^{1}\right)$, for $i=0,1$. Then

$$
A_{0}^{1}, A_{0}^{2}, A_{1}^{1}, A_{1}^{2} \subseteq W^{s}\left(p_{1}, p_{2}\right) .
$$

By symmetry, we also have

$$
-A_{0}^{1},-A_{0}^{2},-A_{1}^{1},-A_{1}^{2} \subseteq W^{s}\left(p_{1}, p_{2}\right) .
$$

Further, let

$$
A_{00}=F_{3}^{-1}\left(-A_{1}^{1}\right), A_{01}=F_{3}^{-1}\left(-A_{0}^{1}\right), A_{10}=F_{3}^{-1}\left(-A_{1}^{2}\right), A_{11}=F_{3}^{-1}\left(-A_{0}^{2}\right) ;
$$

we have $A_{i j} \subseteq W^{s}\left(p_{1}, p_{2}\right)$ for $i, j=0,1$.

This process can be continued indefinitely: each time some interior segment (i.e., interval) of a segment is removed because that interior segment is (eventually) mapped into $W^{s}\left(p_{1}, p_{2}\right)$. Details are omitted. We thus obtain

$$
W^{s}\left(p_{1}, p_{2}\right) \cap\left[v^{*}, v^{*}+x^{*}\right]=\left[v^{*}, v^{*}+x^{*}\right] \backslash \mathcal{C},
$$

where $\mathcal{C}$ is a Cantor-like set.

If, instead of (4.20), we have

$$
\eta_{2}<F_{1}\left(-v^{*}-x^{*}\right)<v_{1}^{*}<\eta_{1}<v^{*}<\eta \text {, i.e., } 3.4172<\alpha<3.5746,
$$

then we need to modify the arguments used between (4.20) and (4.21). This can be done in a similar way. Again we conclude (4.21). Details are omitted.

Case (iii) $n(\alpha)=2, \quad 3.7444<\alpha<3.9759$

Discussion can proceed by considering the following subcases:

(a) $F_{1}\left(-v^{*}-x^{*}\right)<\eta_{3}<v_{2}^{*}<\eta_{2}<v_{1}^{*}<\eta_{1}<v^{*}<\eta$, i.e., $3.9704<\alpha<3.9759$;

(b) $\eta_{3}<F_{1}\left(-v^{*}-x^{*}\right)<v_{2}^{*}<\eta_{2}<v_{1}^{*}<\eta_{1}<v^{*}<\eta$, i.e., $3.7444<\alpha<3.9704$.

The work is similar to Case (ii). Again we conclude

$$
W^{s}\left(p_{1}, p_{2}\right) \cap\left[v^{*}, v^{*}+x^{*}\right]=\left[v^{*}, v^{*}+x^{*}\right] \backslash \mathcal{C}
$$

for a Cantor-like set $\mathcal{C}$.

Case (iv) $n(\alpha)=3, \quad 3.9759<\alpha<4.1415$

Here, we restrict ourselves to $\alpha: 3.9759<\alpha<4$. Then the arguments can be made just as in Cases (ii) and (iii), and we conclude (4.22).

Subtotaling what we have established in Theorems 4.1-4.5 and in the arguments for Cases (i)-(iv), we have classified (or conjectured) the behavior of $F$ for all 


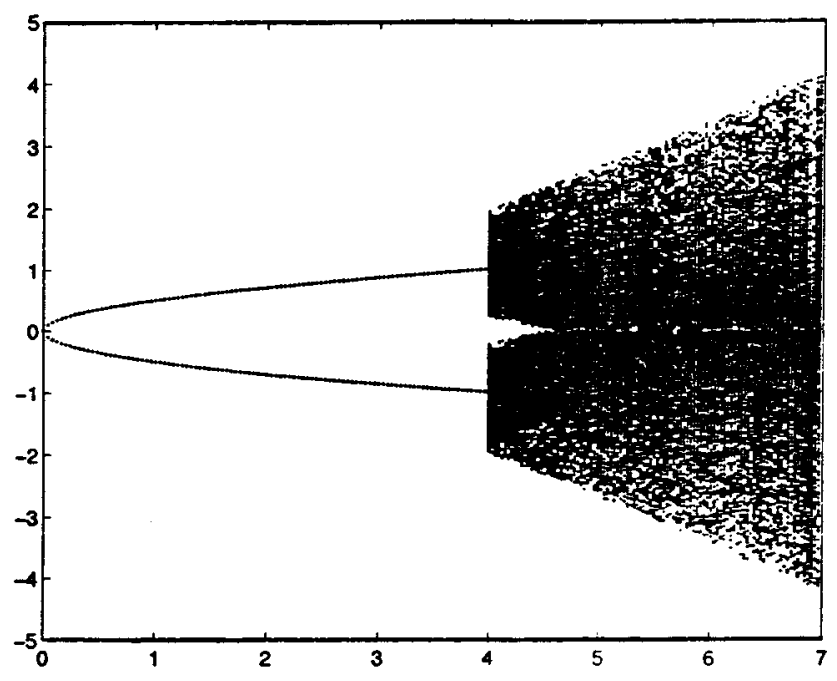

Figure 7 . The orbit diagram of $F$ with respect to the parameter range $0<\alpha<7$.

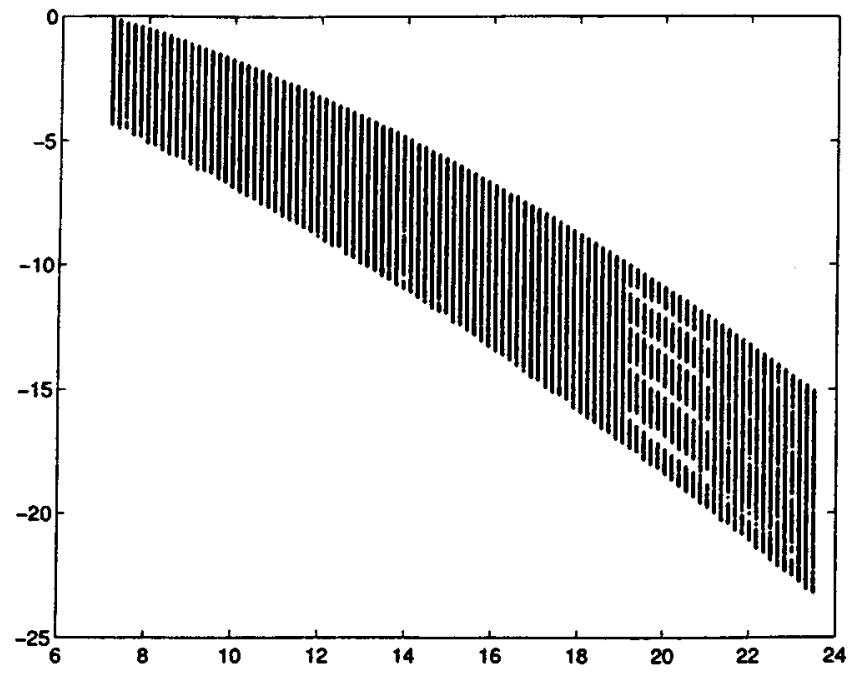

Figure 8 . The orbit diagram of $F$ with respect to the parameter range $7<\alpha<23.5$.

$\alpha>2.9312$. Theorem 3.1 has already characterized the behavior for $\alpha$ : $0<\alpha \leq 1$. The only remaining untreated range of $\alpha: 1<\alpha \leq 2.9312$ is given below.

Theorem 4.6. Let $\alpha \in(1,2.9312)$ and $\beta>0$. Then the curve $u=F(v)$ intersects the line $u=v$ at exactly two points $(\tilde{v},-\tilde{v})=(\tilde{v}, F(\tilde{v}))$ and $(-\tilde{v}, \tilde{v})=(-\tilde{v}, F(-\tilde{v}))$. The set $\{\tilde{v},-\tilde{v}\}$ is the unique period-2 orbit which globally attracts every point $v \in \mathbf{R}$ except the fixed point $v=0$.

Proof. The arguments can be made by considering several cases. Since the work is tedious and mostly straightforward, we omit the details. 


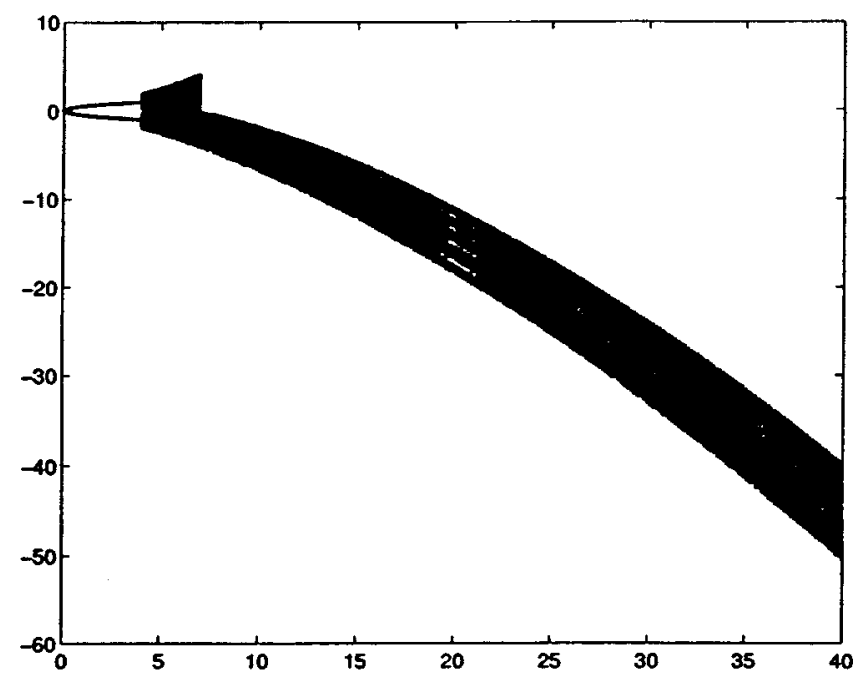

FiguRE 9. The orbit digram with respect to the parameter range $0<\alpha<40$. Because of the symmetry w.r.t. $v=0$, here we have plotted mainly those points $v<0$.

To further support the classification of behavior of the iterates of $F$ with respect to the parameter $\alpha$ (with $\beta$ being normalized to 1 ), we have included three orbit diagrams in Figs. 7, 8 and 9, corresponding, respectively, to the parameter ranges $0<\alpha<7,7<\alpha<23.5$, and $0<\alpha<40$. These diagrams illustrate the asymptotic trajectories of $F^{n}$ for large $n$.

\section{Chaotic Behavior of the Gradient $\left(\mathbf{w}_{\mathbf{x}}, \mathbf{w}_{\mathbf{t}}\right)$ in the Sense of Topological Conjugacy}

We are now able to say in even more precise terms what we mean by chaotic vibrations: the nonlinear boundary condition (1.7) with the hysteretic reflection curve as specified in $\S 2$ causes chaos in the gradient $\left(w_{x}, w_{t}\right)$ in the following sense of topological conjugacy.

From (1.12) and the reflection of characteristics as shown in Fig. 1, we easily derive the following:

$$
\begin{aligned}
w_{x}(1, t+1) & =u(1, t+1)+v(1, t+1) \\
& =F(v(1, t+1))+u(0, t) \\
& =F(v(0, t))+u(1, t-1) \\
& =u(1, t-1)+F(u(1, t-1)) \\
(5.1) \quad & =\frac{1}{2}\left[w_{x}(1, t-1)+w_{t}(1, t-1)\right]+F\left(\frac{1}{2}\left[w_{x}(1, t-1)+w_{t}(1, t-1)\right]\right),
\end{aligned}
$$

for $t \geq 1$. Similarly,

$$
\begin{aligned}
w_{t}(1, t+1)= & -\frac{1}{2}\left[w_{x}(1, t-1)+w_{t}(1, t-1)\right] \\
& +F\left(\frac{1}{2}\left[w_{x}(t, t-1)+w_{t}(1, t-1)\right]\right), \quad t \geq 1 .
\end{aligned}
$$


We may write the above as a $2 \times 2$ iterative system:

$$
\left[\begin{array}{c}
\xi_{n+1} \\
\eta_{n+1}
\end{array}\right]=\left[\begin{array}{c}
\frac{1}{2}\left(\xi_{n}+\eta_{n}\right)+F\left(\frac{1}{2}\left(\xi_{n}+\eta_{n}\right)\right) \\
-\frac{1}{2}\left(\xi_{n}+\eta_{n}\right)+F\left(\frac{1}{2}\left(\xi_{n}+\eta_{n}\right)\right)
\end{array}\right],
$$

where

$$
\begin{aligned}
& \xi_{n}=w_{x}\left(1,2 n+t_{0}\right), \quad \eta_{n}=w_{t}\left(1,2 n+t_{0}\right), \\
& n \geq 1, \quad t_{0} \in[0,2) ; t_{0} \text { is arbitrary but fixed, }
\end{aligned}
$$

with

$$
\begin{gathered}
\xi_{0}=w_{x}\left(1, t_{0}\right)= \begin{cases}F\left(v_{0}\left(1-t_{0}\right)\right)+v_{0}\left(1-t_{0}\right), & 0 \leq t_{0}<1, \\
F\left(u_{0}\left(t_{0}-1\right)\right)+u_{0}\left(t_{0}-1\right), & 1 \leq t_{0}<2 ;\end{cases} \\
\eta_{0}=w_{t}\left(1, t_{0}\right)= \begin{cases}F\left(v_{0}\left(1-t_{0}\right)\right)-v_{0}\left(1-t_{0}\right), & 0 \leq t_{0}<1, \\
F\left(u_{0}\left(t_{0}-1\right)\right)-u_{0}\left(t_{0}-1\right), & 1 \leq t_{0}<2,\end{cases} \\
u_{0}(x) \equiv \frac{1}{2}\left[w_{x}(x, 0)+w_{t}(x, 0)\right]=\frac{1}{2}\left[w_{0}^{\prime}(x)+w_{1}(x)\right], 0 \leq x \leq 1,
\end{gathered}
$$

We now show that the iterative map (5.3) is topologically conjugate to the scalar iterative map $v_{n+1}=F\left(v_{n}\right)$.

Define a set

$$
\mathcal{C}=\left\{(\xi, \eta) \in \mathbf{R}^{2} \mid \xi=c+F(c), \eta=-c+F(c), c \in \mathbf{R}\right\} .
$$

Then $\mathcal{C}$ is a "broken curve" in $\mathbf{R}^{2}$ : at $c= \pm v^{*}$, the mapping

$$
\mathcal{I}: \mathbf{R} \rightarrow \mathbf{R}^{2}, \mathcal{I}(c) \equiv(c+F(c),-c+F(c)),
$$

has discontinuities if $\alpha>1$. Define

$$
\mathcal{F}: \mathbf{R}^{2} \rightarrow \mathbf{R}^{2}, \quad \mathcal{F}\left[\begin{array}{l}
\xi \\
\eta
\end{array}\right]=\left[\begin{array}{r}
\frac{1}{2}(\xi+\eta)+F\left(\frac{1}{2}(\xi+\eta)\right) \\
-\frac{1}{2}(\xi+\eta)+F\left(\frac{1}{2}(\xi+\eta)\right)
\end{array}\right]
$$

Then the iterative system (5.3) can be written as

$$
\left[\begin{array}{l}
\xi_{n+1} \\
\eta_{n+1}
\end{array}\right]=\mathcal{F}\left[\begin{array}{l}
\xi_{n} \\
\eta_{n}
\end{array}\right], \quad n=0,1,2, \ldots
$$

For $n=0,1,2, \ldots$, we easily see that $\left(\xi_{n}, \eta_{n}\right) \in$ range $\mathcal{F}$. Therefore the two dimensional map $\mathcal{F}(5.6)$ is actually "one dimensional":

$$
\left.\widetilde{\mathcal{F}} \equiv \mathcal{F}\right|_{\mathcal{C}}: \mathcal{C} \longrightarrow \mathcal{C} .
$$

We now establish a homeomorphism between the graph of $F$, i.e.,

$$
G(F)=\{(v, F(v)) \mid v \in \mathbf{R}\}
$$

and $\mathcal{C}$ by

$$
Q: \mathbf{R}^{2} \rightarrow \mathbf{R}^{2}, \quad Q\left[\begin{array}{l}
x \\
y
\end{array}\right]=\left[\begin{array}{r}
x+y \\
-x+y
\end{array}\right] .
$$

Then $Q$ is an orthogonal transformation in $\mathbf{R}^{2}$, and

$$
\left.\widetilde{Q} \equiv Q\right|_{G(F)}: G(F) \stackrel{\text { homeomorphism }}{\longrightarrow} \mathcal{C} .
$$


Define

$$
\begin{aligned}
& H: G(F) \longrightarrow G(F), \\
& H\left[\begin{array}{c}
v \\
F(v)
\end{array}\right] \equiv\left[\begin{array}{c}
F(v) \\
F(F(v))
\end{array}\right], \quad v \in \mathbf{R} .
\end{aligned}
$$

That is to say, $H$ is the diagonal operator

$$
H=\left[\begin{array}{cc}
F(\cdot) & 0 \\
0 & F(\cdot)
\end{array}\right]
$$

Using (5.5)-(5.9), it is straightforward to verify that the following diagram is commutative:

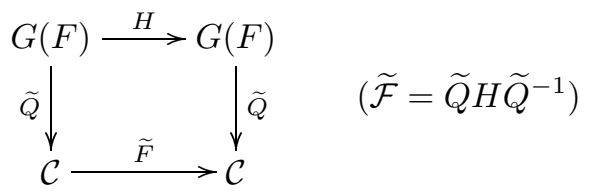

Therefore $\widetilde{\mathcal{F}}$ is topologically conjugate ([7], p. 47]) to a diagonal mapping $H$ both of whose diagonal entries are chaotic for $\alpha$ lying in a certain range. It is in the sense of the topological conjugacy (5.11) that the gradient $\left(w_{x}(1, t), w_{t}(1, t)\right)$ (which is a Poincaré section of $\left.\left(w_{x}(x, t), w_{t}(x, t)\right)\right)$ mapping (5.3) is chaotic (when (5.10) is chaotic).

Of course, it is also possible to prove that (5.5) is a chaotic two dimensional map ([7, Chap. 2]), but the work is more involved.

It is interesting to note that the trajectory $w(x, t)$ is not chaotic (in contrast to the results in Holmes and Marsden [10]), as can be seen from the graphics in the next section, Figs. 18 and 21.

\section{Numerical Simulation And Visualization}

We offer a few computer graphics in this section to visualize the dynamic behavior of the solutions of the PDEs.

We determine the solution $(v(x, t), u(x, t))$ of $(1.13)-(1.16)$ subject to the hysteretic reflection relation (1.19) by the method of characteristics. We have found the following: for $(x, t), 0 \leq x \leq 1, t=2 k+\tau, k=0,1,2, \ldots, 0 \leq \tau<2$,

$$
\begin{aligned}
& v(x, t)= \begin{cases}F^{k}\left(v_{0}(x-\tau)\right), & \tau \leq x, \\
F^{k}\left(u_{0}(\tau-x)\right), & x<\tau \leq 1+x, \\
F^{k+1}\left(v_{0}(2-\tau+x)\right), & 1+x<\tau \leq 2,\end{cases} \\
& u(x, t)= \begin{cases}F^{k}\left(u_{0}(x+\tau)\right), & \tau \leq 1-x, \\
F^{k+1}\left(v_{0}(2-x-\tau)\right), & 1-x<\tau \leq 2-x, \\
F^{k+1}\left(u_{0}(x+\tau-2)\right), & 2-x<\tau \leq 2,\end{cases}
\end{aligned}
$$

where $\left(u_{0}, v_{0}\right)$ is the initial condition as in (1.16). Throughout this section, we choose, for $x$ : $0 \leq x \leq 1$,

$$
\left\{\begin{array}{l}
w_{0}(x)=\frac{4}{\pi}(1-\cos \pi x)-\frac{7}{4} x+\frac{7}{8 \pi} \sin 2 \pi x, \\
w_{1}(x)=4 \sin \pi x+3.5 \sin ^{2} \pi x,
\end{array}\right.
$$


resulting in

$$
\left\{\begin{array}{l}
u_{0}(x)=4 \sin \pi x \\
v_{0}(x)=-3.5 \sin ^{2} \pi x
\end{array}\right.
$$

We note that the initial condition (6.4) satisfies the boundary conditions (1.14) and (1.15) at $t=0$. In this sense, we may say that the initial condition (6.4) and the boundary conditions (1.14) and (1.15) are compatible.

Note that once $v(x, t)$ and $u(x, t)$ have been computed from (6.1) and (6.2), we can recover $w(x, t)$ and $w_{t}(x, t)$ by

$$
w(x, t)=\int_{0}^{x}[u(\xi, t)+v(\xi, t)] d \xi, \quad w_{t}(x, t)=u(x, t)-v(x, t) .
$$

We will illustrate two exemplar cases.

Example 6.1. Square waves, $\alpha=1 / 4, \beta=1$; cf. Theorem 3.1.

Nonlinearities are known to be affiliated with pattern formation. In this case, although no chaos can happen, we have found that there is indeed some pattern, namely, a square wave, being formed. See the solution profiles in Figs. 10-13 for $v(x, t)$ and $u(x, t)$, for time durations $t \in[10,12]$ and $t \in[50,52]$. From these figures, one can see that the corners on the square waves become more and more prominent as time becomes large. The solutions $v(x, t)$ and $u(x, t)$ are seen to take approximately two values alternately; these two values are just the two period-2 points of the mapping $u=F(v)$. Such a square wave solution may be regarded as a PDE analogue of the limit cycle solution of the free van der Pol oscillator in the ODE case.

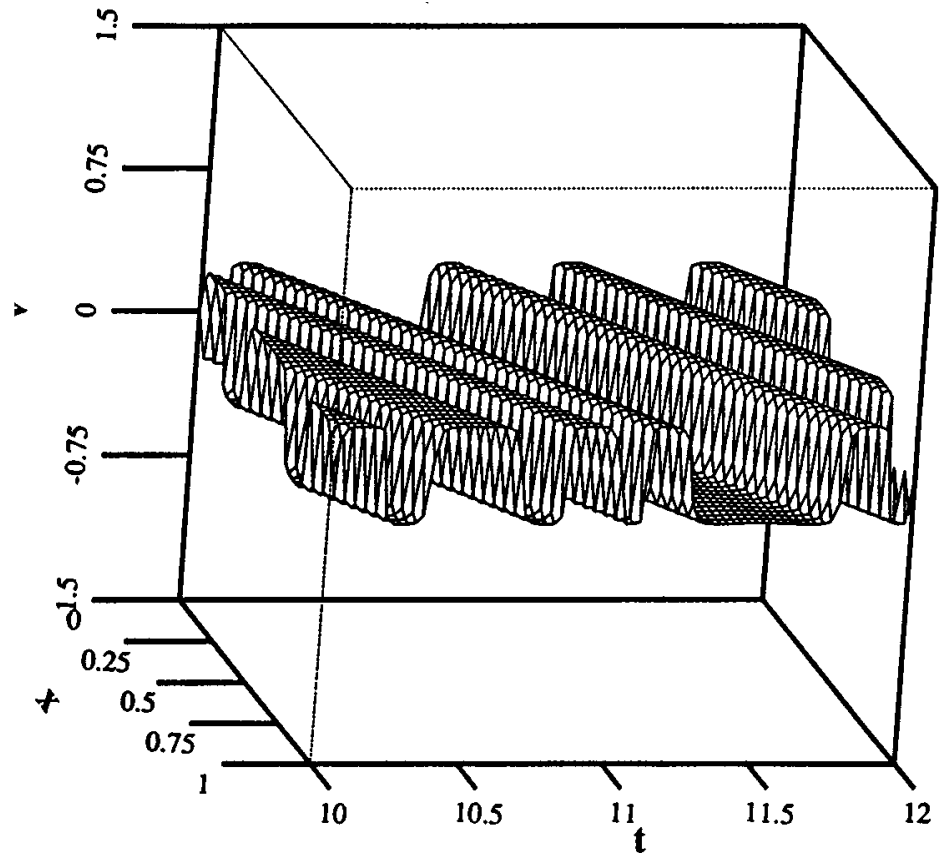

Figure 10. Solution $v(x, t)$ of Example $6.1, t \in[10,12] ; \alpha=$ $1 / 4, \beta=1$ : a square wave. 


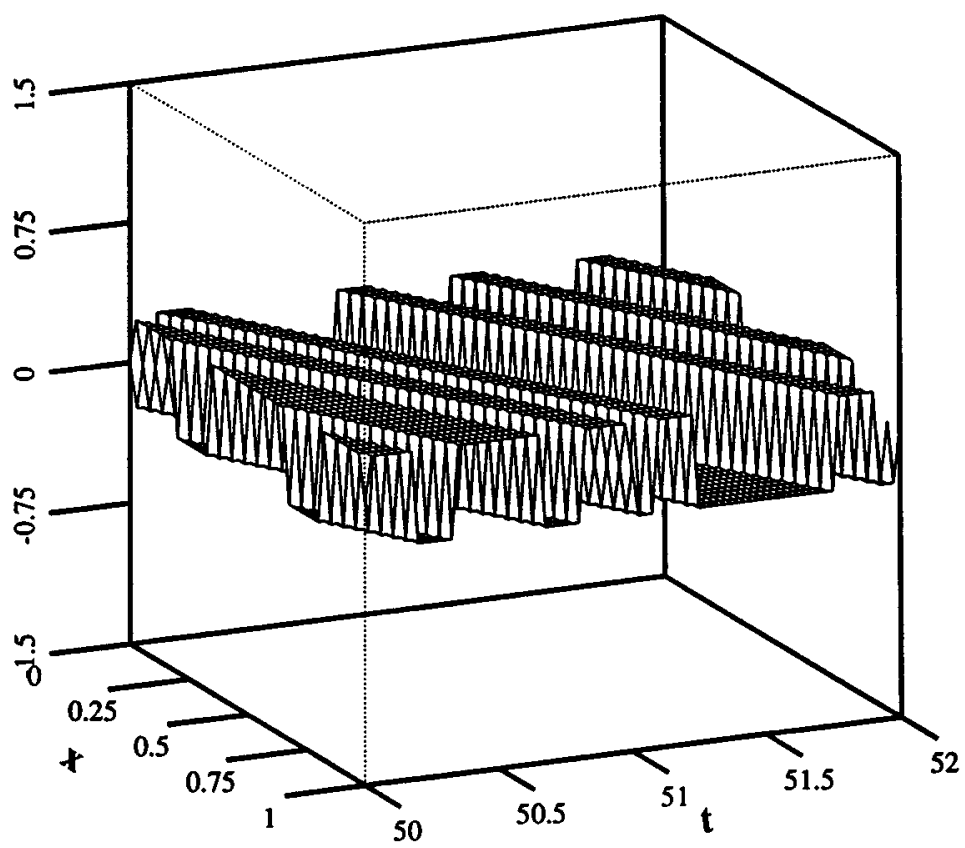

Figure 11. Solution $v(x, t)$ of Example 6.1, $t \in[50,52] ; \alpha=$ $1 / 4, \beta=1$ : a square wave, with steeper corners in comparison with Fig. 10.

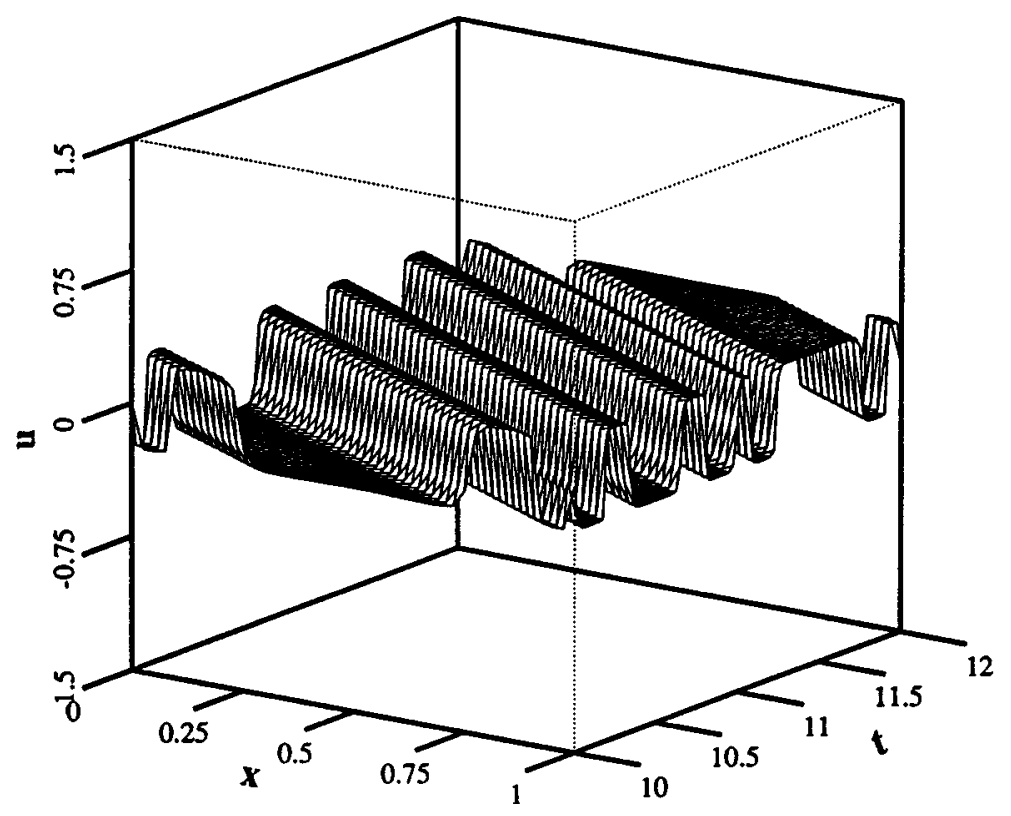

Figure 12. Solution $u(x, t)$ of Example $6.1, t \in[10,12] ; \alpha=$ $1 / 4, \beta=1$ : a square wave. 


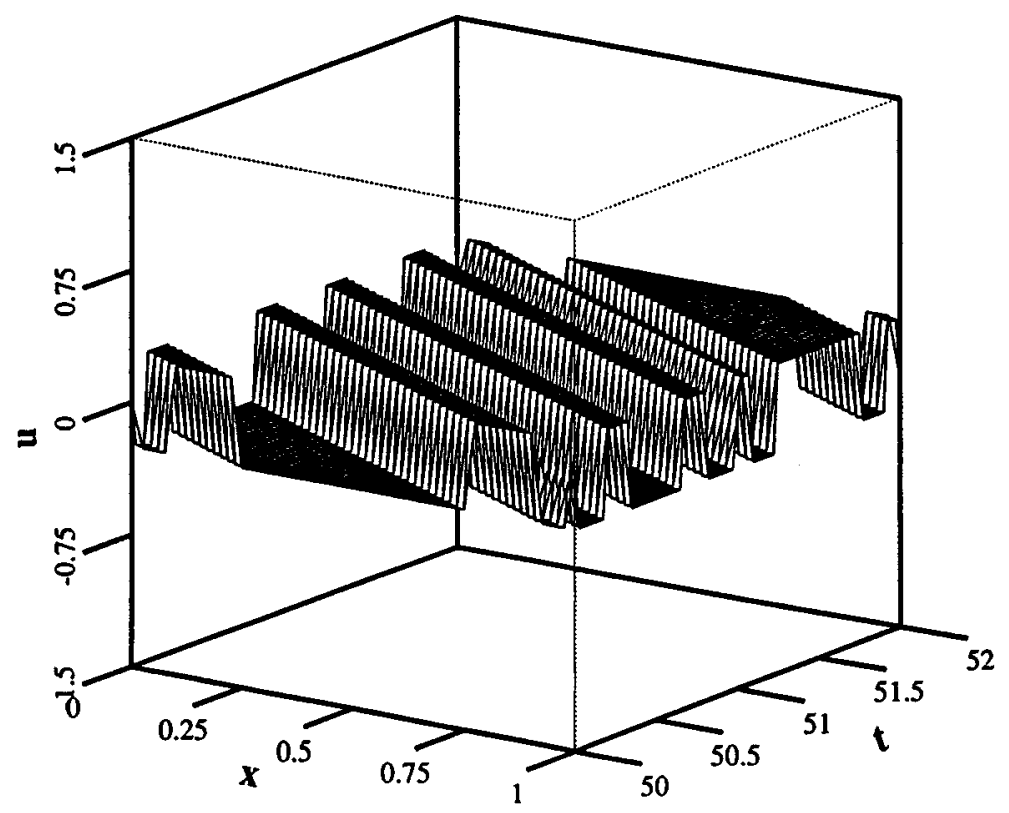

Figure 13. Solution $u(x, t)$ of Example $6.1, t \in[50,52] ; \alpha=$ $1 / 4, \beta=1$ : a square wave,

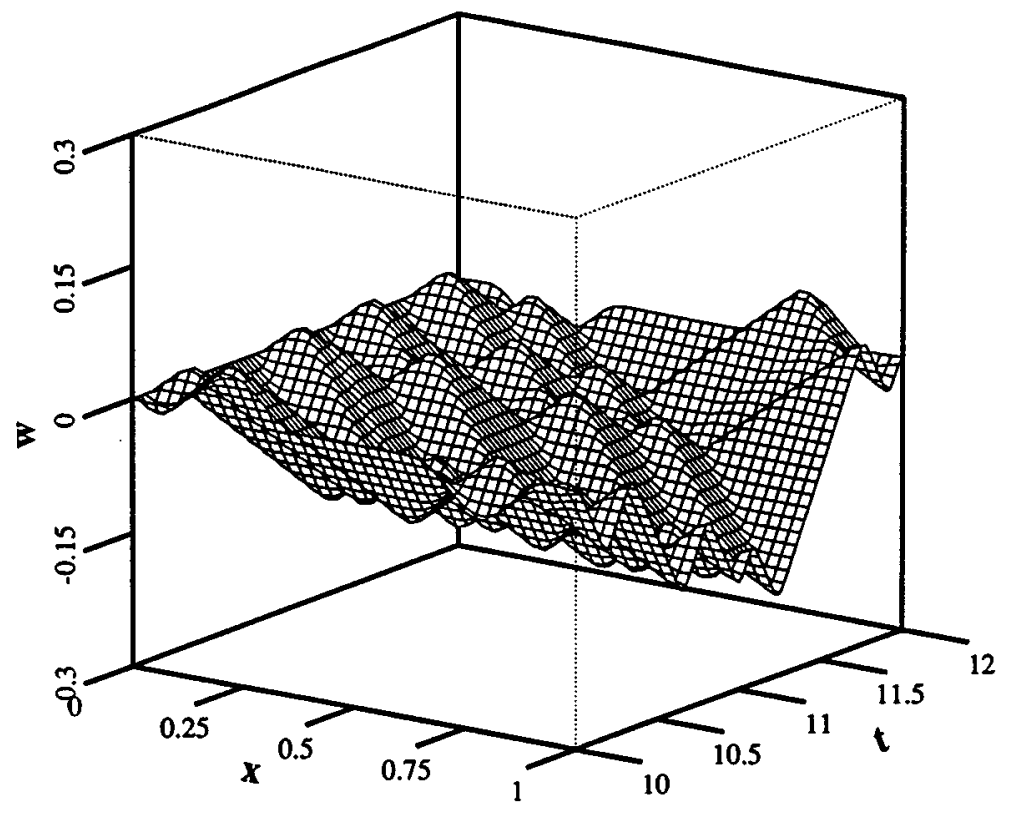

Figure 14. Solution $w(x, t)$ of Example 6.1, $t \in[10,12] ; \alpha=$ $1 / 4, \beta=1$. 


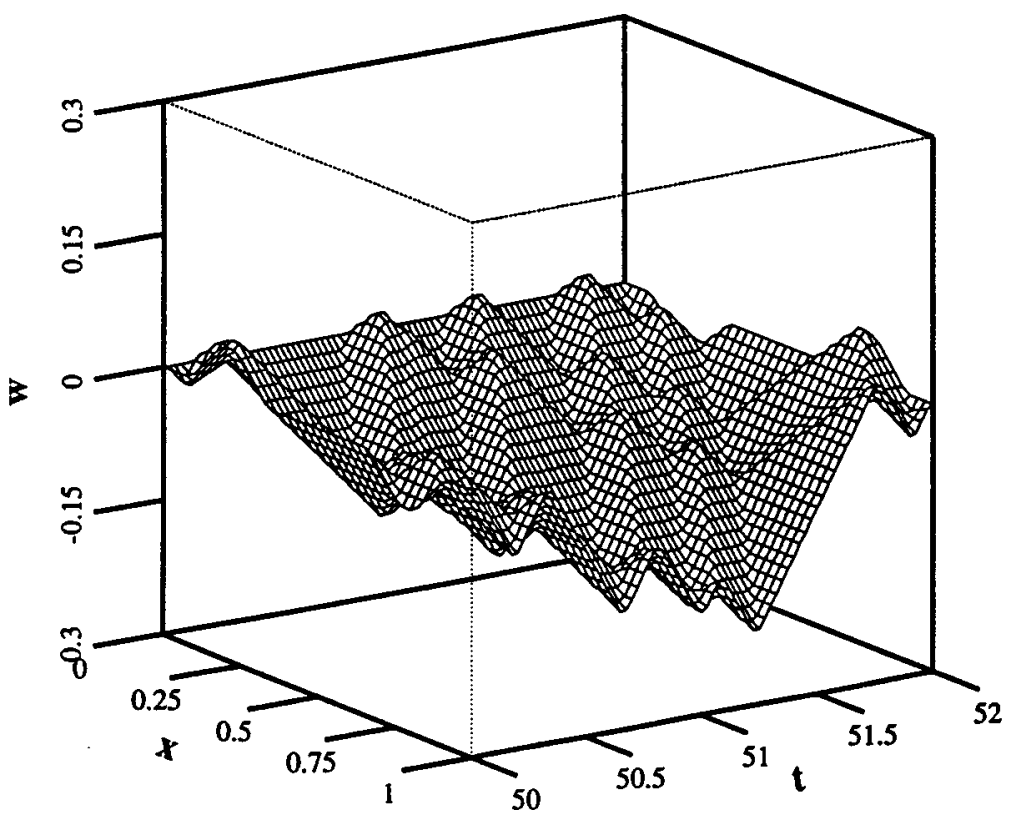

Figure 15. Solution $w(x, t)$ of Example $6.1, t \in[50,52] ; \alpha=$ $1 / 4, \beta=1$.

The displacement function $w(x, t)$ is also shown in Figs. 14 and 15 for $t \in[10,12]$ and $t \in[50,52]$, respectively.

For $\alpha \in(0,2.93120)$, the mapping $F$ has only one repelling fixed point $v=0$, and two globally attracting period-2 points; see Theorem 4.6. Therefore the solution graphics all look similar.

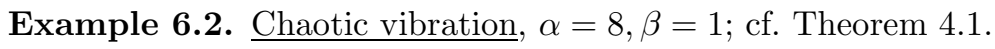

In Figs. 16-18, we plot the graphs of $v(x, t), u(x, t)$ and $w(x, t)$ for the time duration $t \in[10,12]$. Even though $t$ is still not very large, the graphs of $v(x, t)$ and $u(x, t)$ have manifested significant disorder in Figs. 16 and 17.

For $t \in[50,52]$, the graphics are displayed in Figs. 16- 18. Chaotic vibrations have become quite "violent" for $v$ and $u$ in Figs. 19 and 20. "Total chaotic" outlooks of $v$ and $u$, for $t \in[200,202]$, are shown in Figs. 22 and 23.

It is also interesting to observe from Figs. 18 and 21 that the graphics of $w$ seem to manifest some "fractal" outlook, but not chaotic. A more detailed investigation on this is required. 


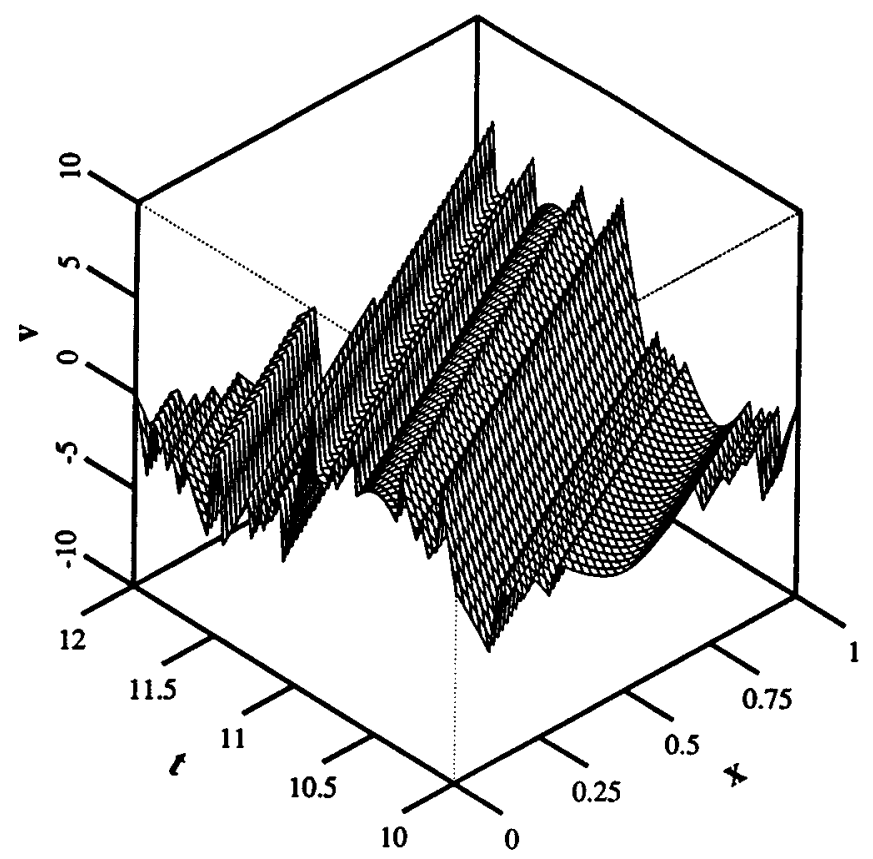

FiguRE 16. Solution $v(x, t)$ of Example 6.2, $t \in[10,12] ; \alpha=$ $8, \beta=1$.

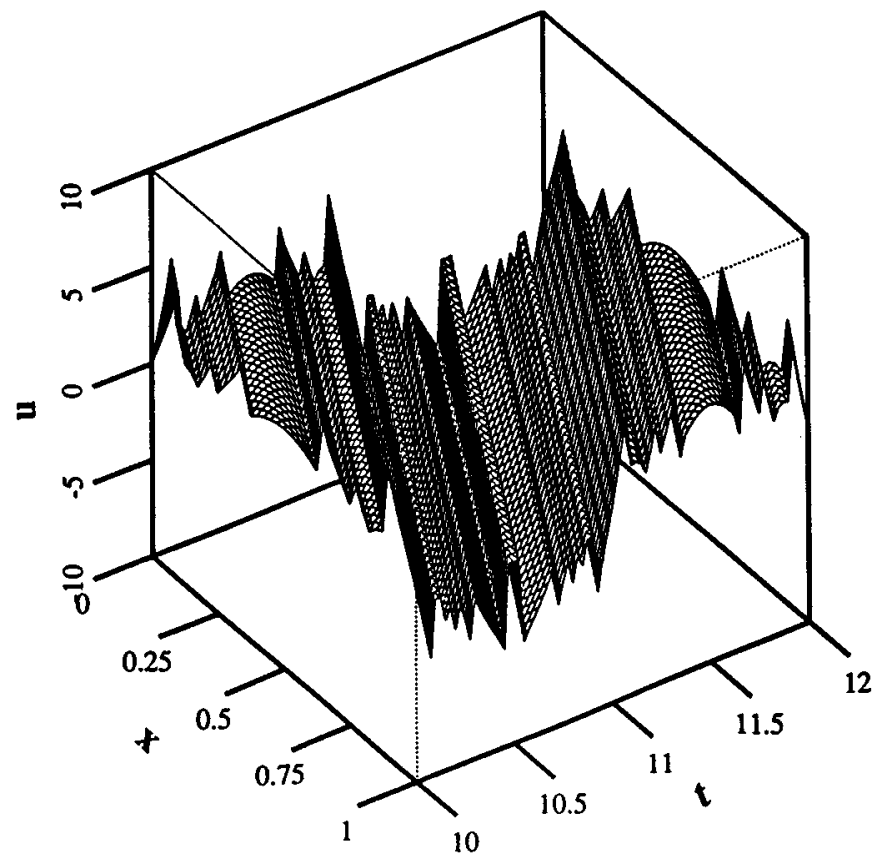

Figure 17. Solution $u(x, t)$ of Example $6.2, t \in[10,12] ; \alpha=$ $8, \beta=1$. 


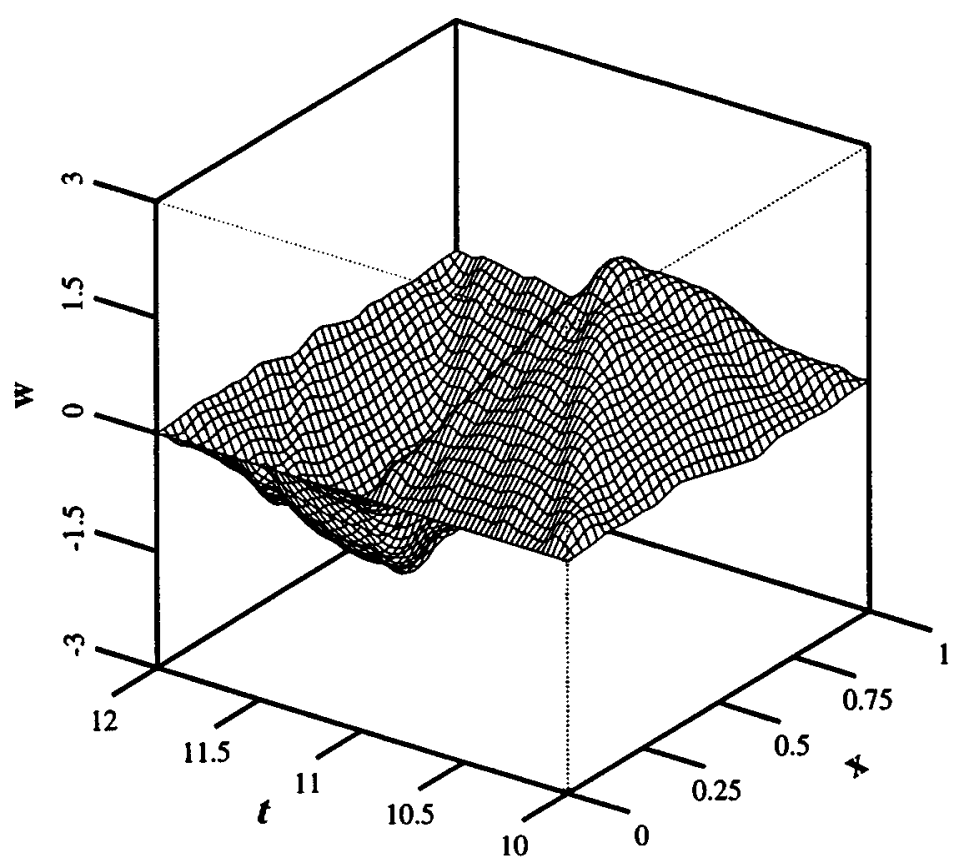

Figure 18. Solution $w(x, t)$ of Example $6.2, t \in[10,12] ; \alpha=$ $8, \beta=1$.

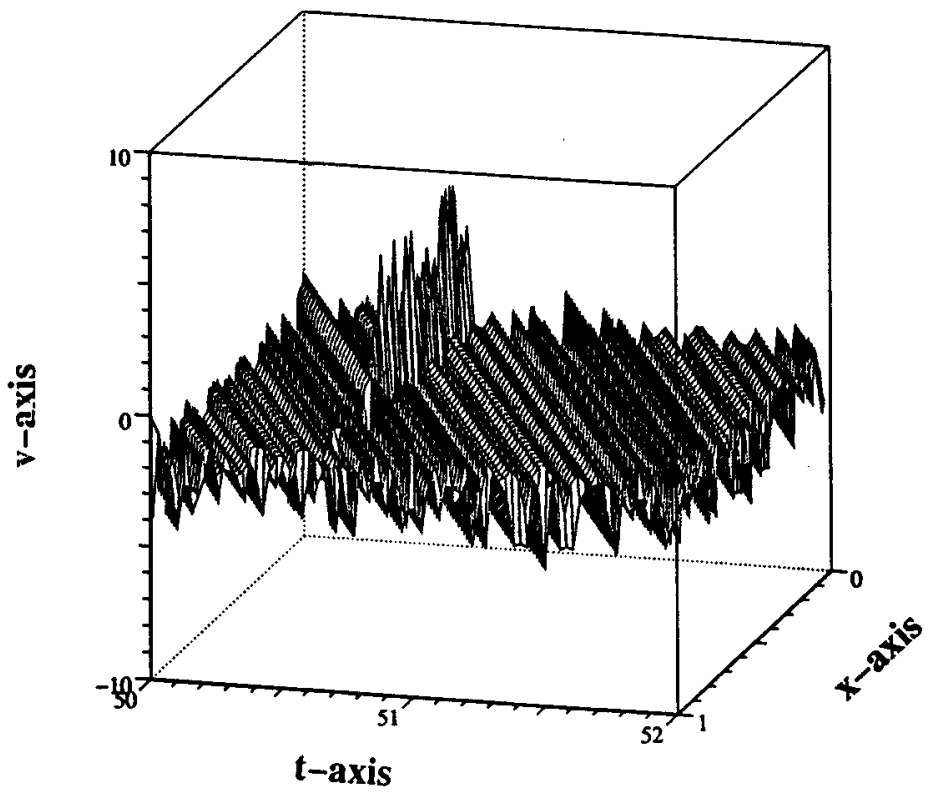

Figure 19. Solution $v(x, t)$ of Example 6.2, $t \in[50,52] ; \alpha=$ $8, \beta=1$. Observe the disorderly vibration of $v$. 


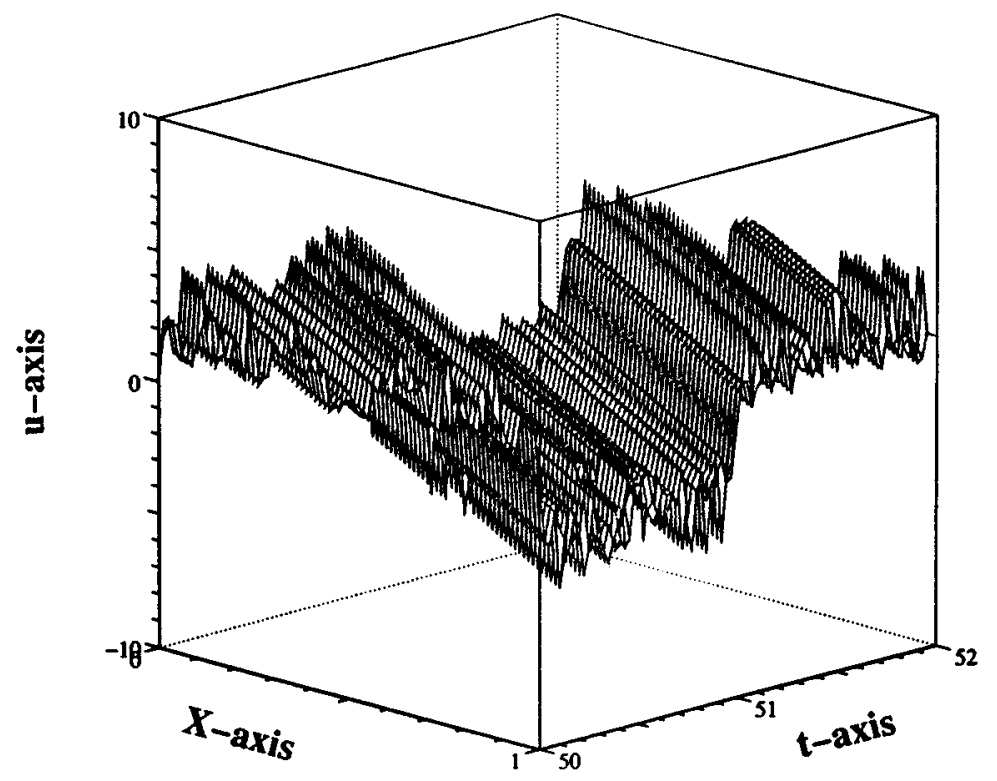

Figure 20. Solution $u(x, t)$ of Example 6.2, $t \in[50,52] ; \alpha=$ $8, \beta=1$. Observe the disorderly vibration of $u$.

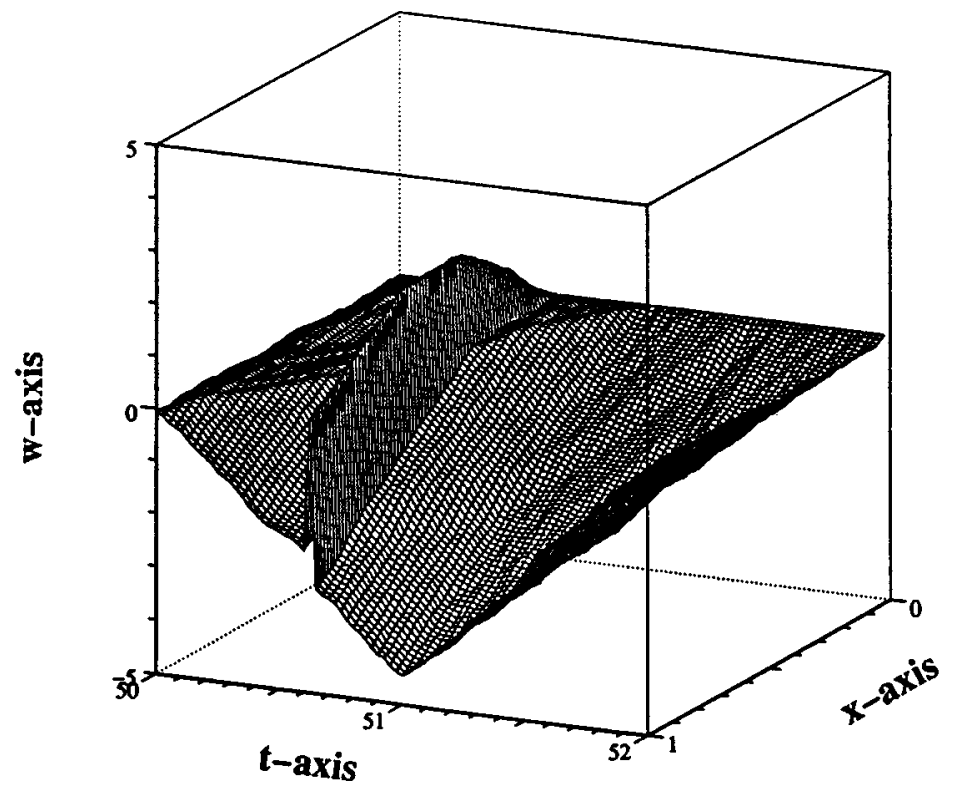

Figure 21. Solution $w(x, t)$ of Example $6.2, t \in[50,52] ; \alpha=$ $8, \beta=1$. Observe the fractal outlook of $w$. 


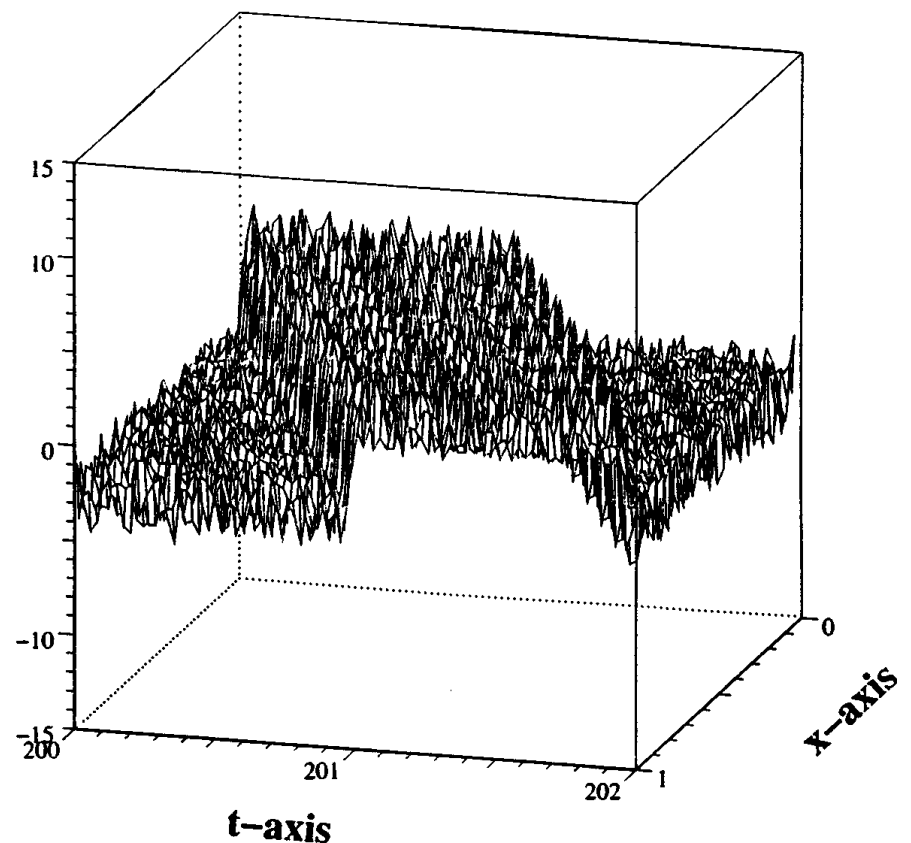

Figure 22. Solution $v(x, t)$ of Example $6.2, t \in[200,202] ; \alpha=8$, $\beta=1$. Observe the total chaotic outlook of $v$.

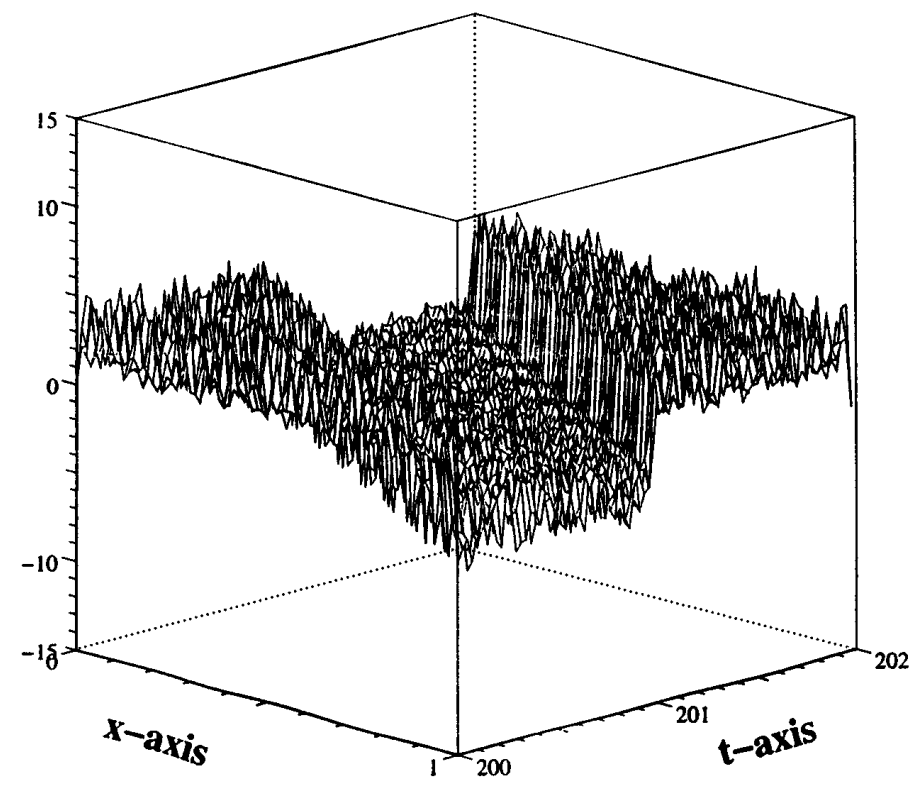

Figure 23. Solution $u(x, t)$ of Example 6.2, $t \in[200,202], \alpha=$ $8, \beta=1$. Observe the total chaotic outlook of $u$. 


\section{Appendix A: Some General Theorems for Chaos by Shift on a Sequence Space for J.P. Keener's Overlapping Case}

We develop some theorems to treat the "overlapping case" of Keener [11, §4], and to apply them to Subsection 4.1 for our own purposes. Consider the mapping

$$
u=F(v), \quad F:[a, b] \longrightarrow[a, b],
$$

(F-I) $\quad F$ has a single jump discontinuity at $v=v^{*}$, where $F\left(v^{*}-\right)=b$, and $F\left(v^{*}\right)=\lim _{v \downarrow v^{*}} F(v)=a$;

(F-II) $\quad F$ is strictly increasing and continuous on $\left[a, v^{*}\right)$ and on $\left[v^{*}, b\right]$;

(F-III) $\quad F$ does not have any fixed point on $(a, b]$;

$(\mathrm{F}-\mathrm{IV}) \quad F(b)>F(a)$;

$(\mathrm{F}-\mathrm{V}) \quad F(b)<v^{*}$.

Now, we mimic and adapt Keener $[11,(1.5)]$ by defining the rotation number $\rho_{I}(v)$ (with respect to a closed subinterval $I \subset[a, b]$ ) for each $v \in[a, b]$ to be

$$
\rho_{I}(v)=\varlimsup_{n \rightarrow \infty} \frac{1}{n} \sum_{k=1}^{n} \chi_{I}\left(F^{n}(v)\right),
$$

where $\chi_{I}$ is the characteristic function of the interval $I$. (The definition of a rotation number was first due to Denjoy [6]. Its properties and physical meanings may be found in Hale [9].) Following [11, Def. 1.2], we define

$$
\text { " } u=F(v) \text { is chaotic if range } \rho_{I} \supseteq[c, d],
$$

$$
\text { for some } c<d \text { and for some subinterval } I \subset[a, b] . "
$$

Since a rational rotation number $\rho_{I}(v)$ corresponds to asymptotically periodic orbits, and an irrational rotation number corresponds to aperiodic trajectories, the iterates $\left\{F^{n}(v) \mid v \in[a, b]\right\}$ on the interval $[a, b]$ are therefore rich in both periodic and aperiodic solutions, each set of which is dense in $I \subseteq[a, b]$. Other definitions of chaos may be found in [1], [7], [13], e.g.

Let $I_{1}$ and $I_{2}$ be two nonempty closed subintervals of $[a, b]$. We say that $F\left(I_{1}\right)$ covers $I_{2}$ if $F\left(I_{1}\right) \supseteq I_{2}$, and write $I_{1} \rightarrow I_{2}$. Let $\left\{I_{j} \mid j=1,2, \cdots, k\right\}$ be a sequence of closed subintervals of $[a, b]$ which are either disjoint or overlap at most at endpoints, s.t.

$$
I_{1} \longrightarrow I_{2} \longrightarrow \cdots \longrightarrow I_{j} \longrightarrow I_{j+1} \longrightarrow \cdots \longrightarrow I_{k}
$$

where $F$ is continuous on each $I_{j}$. We say that (A.4) is a shift on a sequence of intervals. It is known (cf. [11, Corollary 4.2], e.g.) that if the shift sequence (A.4) satisfies

$$
I_{1} \longrightarrow I_{2} \longrightarrow \cdots \longrightarrow I_{j} \longrightarrow I_{j+1} \longrightarrow \cdots \longrightarrow I_{k-1} \longrightarrow I_{k} \longrightarrow I_{j_{0}} \cup I_{1},
$$

where $j_{0} \in\{2, \cdots, k-1, k\}$, then

$$
\text { range } \rho_{I_{1}} \supseteq[c, d]
$$

for some $c<d$. Actually, (A.6) can be slightly improved to the following Lemma A.1.

Note that one can use the above shift sequence to define a transition matrix and obtain a subshift of finite type in symbolic dynamics [7], which may be used to characterize chaos instead of (A.6). 
Lemma A.1. Assume that (A.5) holds for $I_{j} \subseteq[a, b], j=1,2, \cdots, k$, where $F$ is continuous on each $I_{j}$. Then

$$
\text { range } \rho_{I_{1}} \supseteq\left(0, \frac{1}{k}\right] \text {. }
$$

Further, if $F(a)=a$, then range $\rho_{I_{1}} \supseteq\left[0, \frac{1}{k}\right]$.

Since its proof is a straightforward adaptation of that of Keener's [11, Corollary 4.2], we omit it here.

We now define direct preimages of $v^{*}$ by

$$
\left\{\begin{array}{l}
v_{0}^{*} \equiv v^{*}, \\
v_{n+1}^{*}=F^{-1}\left(v_{n}^{*}\right), v_{n+1}^{*} \in\left[a, v^{*}\right), \quad \text { for } n=0,1,2,3, \cdots .
\end{array}\right.
$$

By (F-II), we see that the sequence of such preimages is strictly decreasing:

$$
v_{0}^{*}>v_{1}^{*}>\cdots>v_{j}^{*}>v_{j+1}^{*}>\cdots .
$$

Furthermore, they terminate after finite steps if $F$ is continuously differentiable on $\left[a, v^{*}\right)$ and

$$
F(a)>a, \text { and } F^{\prime}(v) \geq s>0, \text { for some } s, \forall v \in\left[a, v^{*}\right) .
$$

But if the sequence (A.8) does not terminate at a finite place, then, necessarily

$$
\lim _{j \rightarrow \infty} v_{j}^{*}=\tilde{a}, \quad \text { for some } \tilde{a} \geq a .
$$

This happens, e.g., when $\tilde{a}=a$ and $F(a)=a$. These direct preimages form a ladder-like structure as shown in Fig. A.1.

Consider the first case when the sequence (A.8) terminates:

$$
v_{0}^{*}>v_{1}^{*}>v_{2}^{*}>\cdots>v_{k}^{*}, \quad \text { with } v_{k}^{*} \geq a .
$$

We define the following subintervals

$$
\left\{\begin{array}{l}
A_{0}=\left[a, v_{k}^{*}\right), \quad \text { if } v_{k}^{*} \supsetneqq a, \\
I_{1}=\left[v_{k}^{*}, v_{k-1}^{*}\right), \cdots, I_{j}=\left[v_{k-j+1}^{*}, v_{k-j}^{*}\right), \cdots, I_{k}=\left[v_{1}^{*}, v^{*}\right) .
\end{array}\right.
$$

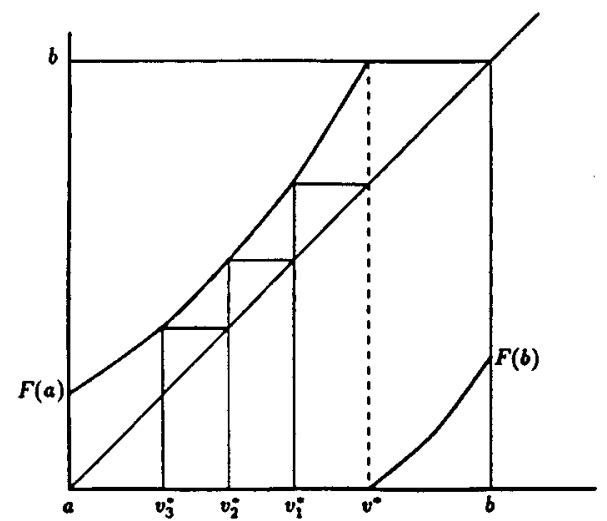

(a)

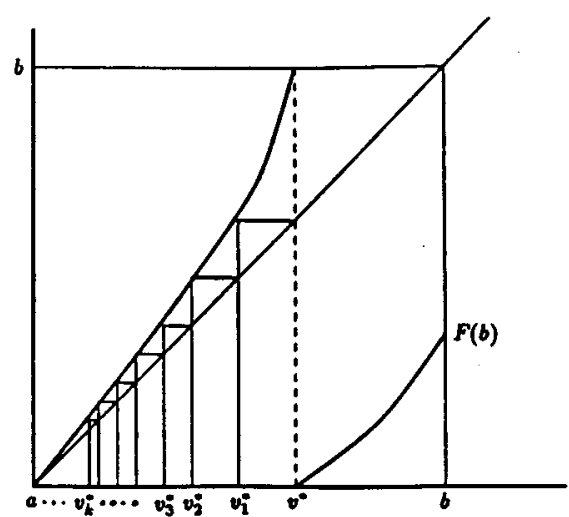

(b)

Figure A.1. Direct preimages $v_{j}^{*}$ : (a) finitely many, (b) infinitely many. 
Then

$$
\left\{\begin{array}{l}
F\left(A_{0}\right) \subseteq I_{1}, \\
F\left(I_{j}\right)=I_{j+1}, \quad \text { for } j=1,2, \cdots, k-1,
\end{array}\right.
$$

and

$$
F\left(I_{k}\right)=\left[v^{*}, v^{*}+x^{*}\right) .
$$

The subintervals in (A.12) are not closed. We slightly modify them by redefining

$$
\widetilde{I}_{j}=\left[v_{k-j+1}^{*}+\varepsilon_{1 j}, v_{k-j}^{*}-\varepsilon_{2 j}\right], \quad j=1,2, \cdots, k,
$$

for some small $\varepsilon_{1 j}, \varepsilon_{2 j}>0$, and simply write

$$
\widetilde{I}_{j}=\left[v_{k-j+1}^{*+}, v_{k-j}^{*-}\right], \quad\left(v_{k-j+1}^{*+} \equiv v_{k-j+1}^{*}+\varepsilon_{1 j}, v_{k-j}^{*-} \equiv v_{k-j}^{*-}-\varepsilon_{2 j}\right) .
$$

Then for appropriately given small $\varepsilon_{1 j}, \varepsilon_{2 j}>0, j=1,2, \cdots, k$, we again have

$$
F\left(\widetilde{I}_{j}\right)=\widetilde{I}_{j+1}, \quad j=1,2, \cdots, k-1 .
$$

Theorem A.1. Assume that $F:[a, b] \rightarrow[a, b]$ s.t. $(F-I)-(F-I I I)$ hold. Consider the sequence of direct preimages $\left\{v_{j}^{*} \mid j=1,2, \cdots\right\}$. Suppose that either

(i) the sequence terminates at a finite place such as (A.11), and $F(b) \geq v_{k-2}^{*}$ for $k$ satisfying (A.11),

or

(ii) the sequence $\left\{v_{j}^{*} \mid j=1,2, \cdots\right\}$ is infinite, and $F(b)>F(a)$.

Then range $\rho_{\tilde{I}_{1}} \supseteq(0, \delta]$ for some $\delta>0$, for some closed interval $\tilde{I}_{1} \subset[a, b]$, and $F$ is chaotic.

Proof. Consider (i). Define

$$
\widetilde{I_{0}}=\left[v^{*}+\varepsilon_{10}, b-\varepsilon_{20}\right]=\left[v^{*+}, b^{-}\right],
$$

for some small $\varepsilon_{10}, \varepsilon_{20}>0$, and let $\widetilde{I}_{j}, j=1, \cdots, k$, be as defined in (A.15) and (A.16). Then we have the shift sequence

$$
\widetilde{I}_{1} \longrightarrow \widetilde{I}_{2} \longrightarrow \cdots \longrightarrow \widetilde{I}_{k} \longrightarrow \widetilde{I}_{0} \longrightarrow \widetilde{I}_{1} \cup \widetilde{I}_{2},
$$

where the first line of the shift sequence above comes from (A.17), while the second line follows from the facts that

$$
F\left(v_{1}^{*}\right)=v^{*}, \quad F\left(v^{*}-\right)=b, \quad F\left(v^{*}\right)=a, \quad F(b) \geq v_{k-2}^{*} .
$$

Therefore we can apply Lemma A.1, and conclude

$$
\text { range } \rho_{\tilde{I}_{1}} \supseteq\left(0, \frac{1}{k+1}\right] \text {. }
$$

Next, consider (ii). Since the sequence $\left\{v_{j}^{*} \mid j=1,2, \cdots\right\}$ is infinite, (A.10) holds. From (A.10), the continuity of $F$ on $\left[a, v^{*}\right)$ and (F-III), we conclude that $\tilde{a}=$ $a=F(a)$; otherwise $\tilde{a} \in\left(a, v^{*}\right)$ is a fixed point of $F$, contradicting (F-III). By assumption that $F(b)>F(a)$, we get

$$
F(b)>F(a)=a ;
$$

therefore from (A.10) we have

$$
F(b) \geq v_{\ell}^{*}, \quad \text { for some } \ell \geq 1 .
$$

Let $k=\ell+2$. Then $F(b) \geq v_{k-2}^{*}$. We now repeat the arguments as in case (i). The proof is complete. 
Remark A.1. (a) Keener's Lemma 4.1 in [11] can now be observed as being identical to our Theorem A.1 part (ii), because in [11] he assumed that $F(a)=a$, implying that the sequence (A.8) is infinite, satisfying (A.10). His "overlapping condition" means $F(b)>F(a)$ in Theorem A.1(ii).

(b) In part (i) of Theorem A.1, the assumption $F(b) \geq v_{k-2}^{*}$ will imply $F(b)>$ $F(a)$, i.e., the case is necessarily "overlapping".

(c) We wish to point out that the closed intervals in the shift sequence as used by Keener must be either totally disjoint or allowed to overlap only at endpoints. Otherwise counterexamples ensue.

\section{Appendix B: M.I.Malkin's Results on the Range of the Renormalized Rotation Number}

M.I. Malkin has done an in-depth investigation of the dynamic behavior of discontinuous maps after Keener [11] and obtained sharp results in [16, 17]. To make our paper sufficiently self-contained, we briefly extract the most relevant ingredients in [17] in order for our use in $\S 4.1$.

Let $I=[a, b]$ and $F: I \longrightarrow I$ such that it has a single jump discontinuity at $v^{*}=v^{*}(F) \in(a, b)$. The map $F$ satisfies the following:

$\left(\mathrm{F}-\mathrm{I}^{\prime}\right) F$ is continuous and monotone increasing on $\left[a, v^{*}\right)$ and $\left(v^{*}, b\right]$, respectively;

$\left(\mathrm{F}-\mathrm{II}^{\prime}\right) \mathcal{D}=\bigcup_{n \geq 0} F^{-n}\left(v^{*}\right)$ is dense in $I$, where $F^{-n}\left(v^{*}\right)=\left\{x \in I \mid F^{n}(x)=v^{*}\right\}$.

We denote the collection of such mappings by $\mathcal{F}(=\mathcal{F}(I))$. We call $F \in \mathcal{F}$ a mapping of Lorenz type on $I$ if the following condition also holds:

$\left(\mathrm{F}-\mathrm{III}^{\prime}\right) \lim _{x \downarrow v^{*}} F(x)=a, \lim _{x \uparrow v^{*}} F(x)=b$.

We use $\mathcal{F}_{L}\left(=\mathcal{F}_{L}(I)\right)$ to denote the set of all mappings of Lorenz type (on the interval $I)$.

Malkin [16] has given several equivalent definitions of topological entropy $h(F)$ for a map $F \in \mathcal{F}$. Here we adopt the following one from him:

$$
h(F)=\text { topological entropy of } F \text { on } I=\lim _{n \rightarrow \infty} \frac{\log \# D\left(F^{n}\right)}{n},
$$

where

$$
\# D\left(F^{n}\right)=\text { the number of discontinuities of } F^{n} \text { on } I=[a, b] .
$$

For given $F \in \mathcal{F}$, let $\rho(F)$ denote the range of rotation numbers:

$$
\rho(F)=\left\{\rho_{I^{\prime}}(v) \mid v \in I\right\}, \text { where } I^{\prime}=\left[v^{*}, b\right] \text { in (A.2). }
$$

For $F \in \mathcal{F}$, there are exactly three possibilities [17, p. 268]:

(i) $\rho(F)$ is a nontrivial closed interval;

(ii) $\rho(F)$ consists of exactly one irrational number;

(iii) $\rho(F)$ consists of exactly one rational number.

Theorem B.1 (Malkin [17, Theorems 1-4]). Let $F \in \mathcal{F}$. Then the following hold:

(a) If $\rho(F)$ is a nontrival closed interval, then $h(F)>0$ and there exists an $M_{0} \in \mathbb{Z}^{+}$s.t. $F$ has periodic points of any period greater than $M_{0}$;

(b) If $\rho(F)$ consists of exactly one irrational number, then $F(a)=F(b)$; 
(c) If $\rho(F)=\left\{\frac{N^{\prime}}{N}\right\}$, where $N, N^{\prime} \in \mathbb{Z}^{+}$are relatively prime, then $F$ has a unique orbit with period $N$, and for some closed interval $J=\left[y_{1}, y_{2}\right] \subseteq I$, we have $\left.F^{N}\right|_{J} \in \mathcal{F}_{L}\left(=\mathcal{F}_{L}(J)\right)$, where $J$ is invariant under $F^{N}, v^{*} \in J$ is the only point of discontinuity of $\left.F^{N}\right|_{J}$, and $y_{1}, y_{2}$ are periodic points of period $N$ of $F$ belonging to the periodic orbit of a certain point $y$ of prime periods $N$ s.t. $y_{1}$ and $y_{2}$ are closest to $v^{*}$ on this $N$-periodic orbit.

According to part (c) of Theorem B.1, because $\left.F^{N}\right|_{J}$ is of Lorenz type, we can determine $\rho\left(F^{N}\right)$ in the renormalized sense on the interval $J$.

From the work in [17], we conclude the following.

Corollary B.1. Let $F \in \mathcal{F}_{L}(I)$ s.t. $h(F)>0$ and $F(a)<F(b)$. Then $\rho\left(F^{k}\right)$ is a nontrivial closed interval for some $k \in \mathbb{Z}^{+}$. Consequently, $F^{k}$ is chaotic in the (renormalized) sense of Keener.

Proof. If $\rho(F)$ is a nontrivial closed interval, then $F$ itself is chaotic in the sense of Keener and we are done.

Therefore, we assume that $\rho(F)$ is not a nontrivial closed interval. By Theorem B.1, $\rho(F)$ consists of either an irrational number or a rational number. But part (b) of Theorem B.1 is ruled out because of the assumption $F(a)<F(b)$. Therefore $\rho(F)=\left\{\frac{N_{0}^{\prime}}{N_{0}}\right\}$ for an irreducible fraction $N_{0}^{\prime} / N_{0}$ by part (c) of Theorem B.1. We consider

$$
f_{1} \equiv F^{N_{0}}: J_{1} \longrightarrow J_{1}, \quad J_{1}=\left[y_{1}^{(1)}, y_{2}^{(1)}\right]
$$

where $y_{1}^{(1)}, y_{2}^{(1)}$ are on the periodic orbit of a certain point $y$

of prime period $N_{0}, y_{1}^{(1)}$ and $y_{2}^{(1)}$ are closest to $v^{*}$ and

$$
v^{*} \in \text { Int } J_{1} \text { (the interior of } J_{1} \text { ). }
$$

Obviously, because of the prime periodicity,

$$
F^{N_{0}}\left(y_{1}^{(1)}\right) \neq F^{N_{0}}\left(y_{2}^{(1)}\right) \text {, i.e., } f_{1}\left(y_{1}^{(1)}\right) \neq f_{1}\left(y_{2}^{(1)}\right) .
$$

If $\rho\left(f_{1}\right)$ is a nontrivial closed interval, then we are done. Otherwise by (B.3), $\rho\left(f_{1}\right)$ consists of exactly an irreducible fraction $N_{1}^{\prime} / N_{1}$. We repeat (B.2) to get a renormalized sequence $f_{2}, f_{3}, \ldots, f_{j}$ :

$$
\rho_{0}=\rho(F)=\left\{\frac{N_{0}^{\prime}}{N_{0}}\right\}, \rho_{1}=\rho\left(f_{1}\right)=\left\{\frac{N_{1}^{\prime}}{N_{1}}\right\}, \ldots, \rho\left(f_{j}\right)=\left\{\frac{N_{j}^{\prime}}{N_{j}}\right\} .
$$

Since $h(F)>0$, the above process terminates for some $j=m \in \mathbb{Z}^{+}$([17, p. 274, line 12]). Therefore $\rho\left(f_{m}\right)$ must be a nontrivial closed interval. Hence

$$
k=N_{0} \cdot N_{1} \cdots N_{m}
$$

makes $\rho\left(F^{k}\right)$ a nontrival closed interval (in the renormalized sense).

Appendix C: Electronic Block Circuit Design Realizing the Piecewise Continuous Hysteretic Reflection Curve $F$

Earlier work on electronic block circuit design having chaotic responses can be found in the work of Baillieul, Brockett and Washburn [2] and Saito [19], e.g. in those papers, the (Poincaré) map $F$ was piecewise linear, continuous ([2]) or with a single jump discontinuity ([19]). 
In our work, we require certain relationship $u=F(v)$ at the right boundary $x=1$, where $u$ and $v$ satisfy (1.18). Rewrite (1.18) as

$$
\begin{aligned}
& (u-v)^{3}+\frac{1-\alpha}{\beta}(u-v)+\frac{2}{\beta} v=0, \\
& u=\left(1+\frac{2}{\alpha-1}\right) v+\frac{\beta}{\alpha-1}(u-v)^{3}, \quad \alpha>1 .
\end{aligned}
$$

We obtain the following block diagram in Fig. C.1, where the forward path, consisting of a linear amplifier $\left(1+\frac{2}{\alpha-1}\right) \cdot$, gives rise to the term proportional to $v$, i.e. $\left(1+\frac{2}{\alpha-1}\right) v$, on the RHS of (C.1). The feedback path consists of a nonlinear device capable of generating a cubic nonlinearity $\frac{\beta}{\alpha-1}(u-v)^{3}$. (In particular, tunnel diodes may be used for this purpose, since their transfer characteristics are often approximated by a cubic law.) The final input-output (I/O) relation is therefore (C.1).

This I/O relation, (C.1), fails to satisfy the mathematical unique solvability condition for $v \mapsto u$ when $|v|<v^{*}$. But knowledge from nonlinear electronic oscillations quickly points out to us that the lack of mathematical uniqueness does not prevent the system from undergoing oscillations-actually, when $|v|<v^{*}$, the system will oscillate according to the natural hysteresis loop as shown in Fig. 6(b). This follows as a consequence of stability, because for $|v|<v^{*}$, the upper and lower branches of the multivalued relation $v \mapsto u$ are stable, while the middle branch is unstable.

Next, we consider an analog circuit design that will have an I/O relation as our controlled hysteretic curve Fig. 7 . We note that the $u=F(v)$ relation is given by (2.20) and (2.22). Since this relation is piecewise defined, for $v<-v^{*},|v|<v^{*}$, and $v>v^{*}$, in principle what we need is a switching circuit. This is provided in Fig. C.2.

$v_{c c}$ : "collector-cutoff" voltage;

$C_{1}$ block: a standard comparator, allowing $v \geq v^{*}$ to pass;

$C_{2}$ block: a "hysteresis comparator", allowing $v:|v|<v^{*}$ to pass;

$C_{3}$ block: a standard comparator, allowing $v \leq-v^{*}$ to pass;

Block Fig. C.1: the block diagram as shown in Fig. C.1;

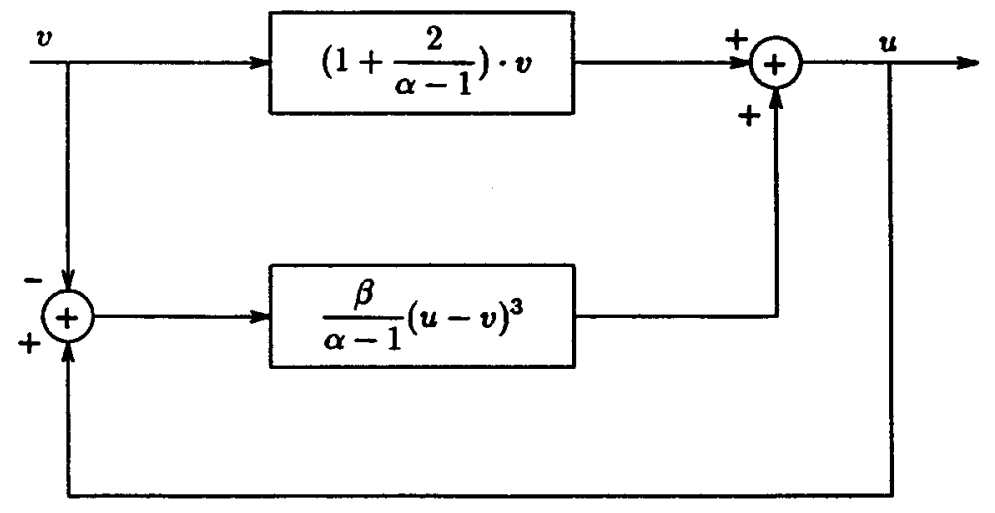

Figure C.1. A nonlinear circuit whose I/O relation satisfies equation (C.1). 


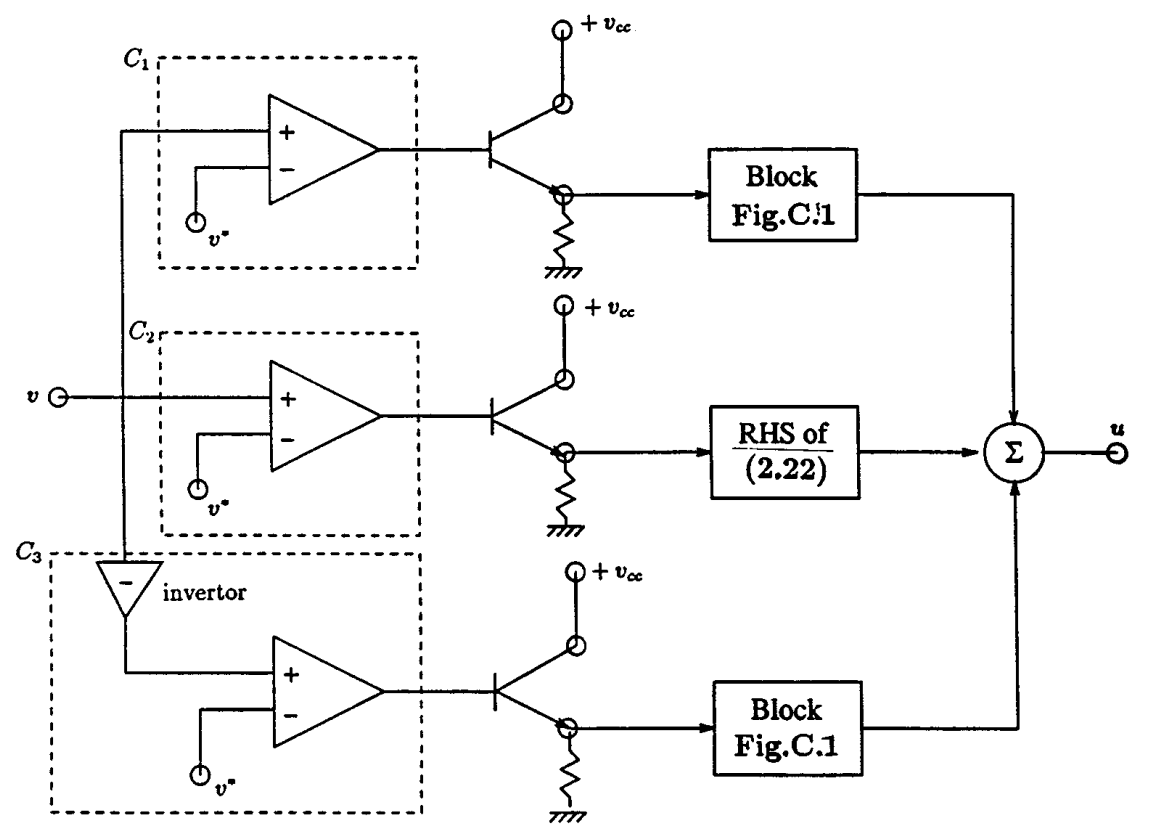

Figure C.2. A switching circuit with I/O relation required as a controlled hysteretic curve.

RHS of (2.20): a combination of nonlinear devices capable of I/O characteristics given by equation (2.20). In practice, however, an I/O curve consisting of a continuous piecewise linear function is often accepted as an alternative or an approximation.

Suppose an electronic device can convert a digital signal $v$ into an analog voltage $v$. Then the circuit design as shown in Fig. C.2 will yield the required I/O response as in our Fig. 7, the hysteretic reflection curve. Note that the design in Fig. C.2 incorporates the block diagram in Fig. C.1 as its parts.

\section{ACKNOWLEDGMENT}

Professor V. Afraimovich has pointed out the relevance of M.I. Malkin's papers $[16,17]$, which enable us to establish Theorem 4.2 in this paper. The reviewer has also offered some constructive criticism. To both of them we express our sincere thanks.

Note added in proof. Further studies of chaotic vibration of the wave equation pertinent to other types of boundary conditions and hysteresis may be found in the following two papers:

1. G. Chen, S. B. Hsu and J. Zhou, Chaotic vibrations of the one-dimensional wave equation due to a self-excitation boundary condition, Part II: energy injection, period doubling and homoclinic orbits, to appear in Int. J. Bifurcation \& Chaos Vol. 8, No. 3, 1998.

2. Ibid., Part III: natural hysteresis memory effects, to appear in Int. J. Bifurcation \& Chaos Vol. 8, No. 3, 1998. 


\section{REFERENCES}

1. J. Banks, J. Brooks, G. Cairns, G. Davis and P. Stacey, On Devaney's definition of chaos, Amer. Math. Monthly 99 (1992), 332-334. MR 93d:54059

2. J. Baillieul, R.W. Brockett, and R.B. Washburn, Chaotic motion in nonlinear feedback systems, IEEE Trans. Circuits Syst. 27 (1980), 990-997. MR 82d:58039

3. G. Chen, S.B. Hsu and J. Zhou, Linear superposition of chaotic and orderly vibrations on two serially connected strings with a van der Pol joint, Int. J. Bifurcation \& Chaos, 6 (1996), $1509-1527$.

4. G. Chen and J. Zhou, Vibration and Damping in Distributed Systems, Vol. I: Analysis, Estimation, Attenuation and Design, CRC Press, Boca Raton, FL, 1993. MR 95a:73030a

5. G.R. Chen and X. Dong, From chaos to order-perspectives and methodologies in controlling chaotic nonlinear dynamical systems, Int. J. Bifurcation \& Chaos, 3 (1993), 1363-1409.

6. A. Denjoy, Sur les courbes définies par les équations différentielles à la surface du tore, J. Math. Pures Appl., (9) 11 (1932), 333-375.

7. R.L. Devaney, An Introduction to Chaotic Dynamical Systems, Addison-Wesley, New York, 1989. MR 91a:58114

8. J. Guckenheimer and P. Holmes, Nonlinear Oscillations, Dynamical Systems, and Bifurcation of Vector Fields, Springer-Verlag, New York 1983. MR 85f:58002

9. J.K. Hale, Ordinary Differential Equations, Wiley, New York, 1969. MR 54:7918

10. P. Holmes and J. Marsden, A partial differential equation with infinitely many periodic orbits: chaotic oscillators of a forced beam, Arch. Rat. Mech. Anal. 76 (1981), 135-165. MR 82m:58039

11. J.P. Keener, Chaotic behavior in piecewise continuous difference equations, Transactions Amer. Math. Soc., 261 (1980), 589-604. MR 81j:58055

12. A. Lasota and J.A. Yorke, On the existence of invariant measures for piecewise monotonic transformations, Transactions Amer. Math. Soc., 186 (1973), 481-488. MR 49:538

13. T.Y. Li and J.A. Yorke, Period three implies chaos, Amer. Math. Monthly, 82 (1975), 985. MR 52:5898

14. T.Y. Li and J.A. Yorke, Ergodic transformations from an interval into itself, Transactions Amer. Math. Soc., 235 (1978), 183-192. MR 56:15883

15. A. Majda, Disappearing solutions for the dissipative wave equation, Indiana Univ. Math. J., 24 (1975), 1119-1133. MR 52:6194

16. M.I. Malkin, On continuity of entropy of discontinuous mappings of the interval, Selecta Math. Sovietica 8(2) (1989), 131-138.

17. M.I. Malkin, Rotation intervals and the dynamics of Lorenz type mappings, Selecta Math. Sovietica 10(3) (1991), 265-275. CMP 91:16

18. F.C. Moon, Chaotic Vibrations, Wiley-Interscience, New York, 1987. MR 89h:58127

19. T. Saito, A chaos generator based on a quasi-harmonic oscillator, IEEE Trans. Circuits Syst., 32 (1985), 320-331. MR 86f:58108

20. J.J. Stoker, Nonlinear Vibrations, Wiley-Interscience, New York, 1950. MR 11:666a

21. S. Wiggins, Introduction to Applied Nonlinear Dynamical Systems and Chaos, SpringerVerlag, New York, 1990. MR 92a:58041

Department of Mathematics, Texas A\&M University, College Station, Texas 77843

E-mail address: gchen@math.tamu.edu

Department of Mathematics, National Tsing Hua University, Hsinchu 30043, Taiwan, R.O.C.

E-mail address: sbhsu@am.nthu.edu.tw

Department of Mathematics, Texas A\& M University, College Station, Texas 77843

E-mail address: jzhou@math.tamu.edu

Department of Electrical Engineering University of Houston, Houston, Texas 772044793

E-mail address: chengr@tree.egr.uh.edu

Department of Environmental Science, University of Milan, Milan I-20126, Italy

E-mail address: giovanni@alpha.disat.unimi.it 